\title{
ON THE SOLUTION OF CONVECTION-DIFFUSION BOUNDARY VALUE PROBLEMS USING EQUIDISTRIBUTED GRIDS*
}

\author{
C. J. BUDD ${ }^{\dagger}$, G. P. KOOMULLIL ${ }^{\ddagger}$, AND A. M. STUART ${ }^{\S}$
}

\begin{abstract}
The effect of using grid adaptation on the numerical solution of model convectiondiffusion equations with a conservation form is studied. The grid adaptation technique studied is based on moving a fixed number of mesh points to equidistribute a generalization of the arc-length of the solution. In particular, a parameter-dependent monitor function is introduced which incorporates fixed meshes, approximate arc-length equidistribution, and equidistribution of the absolute value of the solution, in a single framework. Thus the resulting numerical method is a coupled nonlinear system of equations for the mesh spacings and the nodal values. A class of singularly perturbed problems, including Burgers's equation in the limit of small viscosity, is studied. Singular perturbation and bifurcation techniques are used to analyze the solution of the discretized equations, and numerical results are compared with the results from the analysis. Computation of the bifurcation diagram of the system is performed numerically using a continuation method and the results are used to illustrate the theory. It is shown that equidistribution does not remove spurious solutions present on a fixed mesh and that, furthermore, the spurious solutions can be stable for an appropriate moving mesh method.
\end{abstract}

Key words. convection-diffusion equations, mesh adaptation, equidistribution, continuation

AMS subject classifications. 65M20,65M50, 65N50, 35B40

PII. S1064827595280454

1. Introduction. The solution of boundary value problems (BVPs) using finite difference methods consists of discretizing the differential equations on a finite mesh and solving the system of equations for the values of the functions at these mesh locations. Adaptive meshes are widely utilized for problems containing strong gradients and for problems where important features of the solution develop on small length scales. References for applications in computational fluid dynamics are given in [18] and [12], [13], [14], [20] give more general references for the application and analysis of mesh adaption. Adaptive meshes are essential if accurate and reliable estimates of the solution structure are to be made, and much effort is put into designing suitable meshes for complex geometries. However, the analysis of the reliability of adaptive meshing strategies with regard to giving accurate solutions and rejecting spurious ones is still in its infancy. The fundamental question we ask is this: how well do the qualitative properties of the discrete system with mesh adaption reflect the qualitative properties of the continuous system it is approximating?

Our studies of this question will focus on adaptive numerical discretizations of reaction-convection-diffusion BVPs of conservation form:

$$
\left\{\begin{array}{c}
u_{t}=\varepsilon u_{x x}-f\left(u, u_{x}\right), \\
u(0)=A, \quad u(1)=-A, \quad A>0,
\end{array}\right.
$$

\footnotetext{
* Received by the editors January 20, 1995; accepted for publication (in revised form) January 22, 1997; published electronically September 10, 1998.

http://www.siam.org/journals/sisc/20-2/28045.html

${ }^{\dagger}$ Department of Mathematics, University of Bath, Claverton Down, Bath BA2 7AY, UK (cjb@ maths.bath.ac.uk).

${ }^{\ddagger}$ NRC Associate, Fluid Dynamics Division, NASA Ames Research Center, Moffett Field, CA 94035-1000.

$\S$ Division of Applied Mechanics, Durand 252, Stanford University, Stanford, CA 94305-4040 (stuart@sccm.stanford.edu).
} 
where

$$
f\left(u, u_{x}\right)=F(u)_{x}, \quad F(u)>0 \quad \text { if } \quad u>0 \quad \text { and } \quad F(u)=F(-u) .
$$

We look at both steady states and time-evolving solutions. The steady states satisfy the identity

$$
\varepsilon u_{x}-F(u)=-k / 2,
$$

where $k$ is a suitable constant. We note that even if $f$ is a linear function of $u$, the addition of an adaptive strategy to solve (1.1) typically leads to a nonlinear discrete system.

An important example of such an equation is the viscous Burgers equation where

$$
f\left(u, u_{x}\right)=u u_{x}, \quad F(u)=\frac{u^{2}}{2}
$$

in (1.1), (1.2). This is an important test problem for many methods in computational fluid dynamics and has a unique, monotone decreasing, and symmetric steady solution given by

$$
u(x)=-m \tanh \left(\frac{m\left(x-\frac{1}{2}\right)}{2 \varepsilon}\right),
$$

where $m$ is a constant given by the unique solution of

$$
m \tanh \left(\frac{m}{4 \varepsilon}\right)=A .
$$

This steady solution is a global attractor for the time-dependent problem.

As $F(u)=F(-u)$ the steady state of (1.1) is invariant under the action of the map

$$
u \rightarrow-u, \quad x \rightarrow 1-x .
$$

All continuous steady solutions of (1.1) under (1.2) are necessarily symmetric and satisfy

$$
u(x)=-u(1-x)
$$

so that

$$
u(1 / 2)=0 .
$$

If $\varepsilon$ is small then there is a sharp transition layer in the solution where $u(x)$ changes sign. This layer is centered at $x=\frac{1}{2}$ and is of $\mathcal{O}(\varepsilon)$ in width. Outside the transition layer $|u|$ is close to $A$.

In contrast, when $\varepsilon=0$, the resulting inviscid equation has many steady weak solutions which satisfy $u_{x}=0, F(u)=F(A)$ and are given by

$$
u(x)=A \quad \text { if } \quad x<x_{0}, \quad u(x)=-A \quad \text { if } \quad x \geq x_{0} .
$$

For these solutions the transition layer is at $x_{0}$, where $x_{0}$ is arbitrary. Only one of these is the weak limit of a viscous solution. However, exponentially small (in $\varepsilon$ ) 
changes in the symmetry of the boundary conditions of the viscous problem can lead to solutions with significantly displaced transition layers. This phenomenon is referred to as supersensitivity in the papers of [9], [10], [11], and [19]. For the time-dependent problem, if $\varepsilon$ is small, a solution will rapidly evolve to one with $u_{x}$ close to zero almost everywhere. This solution, again, has a thin transition layer of width $\mathcal{O}(\varepsilon)$, where $u$ changes sign, which is centered at a point determined by the initial conditions. Over much longer time scales this transition layer moves slowly towards the point $x=1 / 2$ and so the solution converges to the symmetric steady state. This phenonenon is often called metastability [3].

When a method using a fixed mesh at points $x_{j}=j / J, j=0, \ldots, J$ (not adapted to the solution) is used to find the steady states of (1.1) it is well known [6] here that this may give rise to spurious solutions. Such solutions frequently place the transition layers in an incorrect position. Typically, however, when working with problems that have sharp transition layers, an adaptive strategy is used which places mesh points $x_{j}$ into regions where the computed solution has a high gradient. Such strategies are hoped to produce reliable answers and to preserve qualitative properties of the solution such as symmetry and monotonicity. In this paper we consider strategies which move a fixed number of mesh points so as to equidistribute a positive monitor function of the solution. A natural question to ask is: can such an adaptive stategy for finding the steady solutions of (1.1) place the transition layer in the correct position and give monotone solutions? Briefly, the answer to this question is "no, in general." Indeed, we show that even when the transition layer is well resolved by the mesh (so that many points are moved into the region where $u$ changes sign), it can still appear in entirely the wrong position.

To demonstrate this we solve for the steady state of (1.1) using an adaptive strategy in which a fixed number of $J$ mesh points $x_{j}$ are placed in such a manner that a generalization of the the arc-length of the solution is equidistributed over each interval $\left[x_{j}, x_{j+1}\right]$. If $\varepsilon$ is sufficiently large compared with $1 / J$, then the numerical method has a unique, monotone, symmetric solution with a transition layer at $x=1 / 2$. However, as $\varepsilon$ is reduced then asymmetric (spurious) solutions bifurcate from this branch when $\varepsilon=\varepsilon_{S B}$. Close to $\varepsilon_{S B}$ then both the true and spurious solutions are monotone decreasing in $x$. These asymmetric solutions persist as $\varepsilon$ is reduced. Further asymmetric solutions arise at subsequent symmetry breaking bifurcations from the main branch. For small $\varepsilon$ these asymmetric solutions are perturbations of the many weak solutions arising in the continuous problem when $\varepsilon=0$, and they exhibit transition layers placed almost arbitrarily in the interval $[0,1]$. Similar behavior (including the existence of a related value $\varepsilon_{S B}$ ) is observed for the case of a uniform grid. Thus a unique numerical solution which correctly places the transition layer exists only if $\varepsilon>\varepsilon_{S B}$. We make some estimates of $\varepsilon_{S B}$ and show that its value is not significantly reduced by using the arc-length-based adaptive strategy. However, we show that if a strategy is used which ensures that the estimate $\left|u_{j+1}-u_{j}\right|$ for $\left|u_{x}\right|$ is equidistributed by the mesh, then the resulting solution is both monotone decreasing and symmetric for all values of $\varepsilon$. However, such an equidistribution method is typically not robust and will not perform well in some computations due to the nonsmoothness of the monitor function.

As a second investigation we look at the time-dependent problem (1.1) using an adaptive method which moves the mesh points $x_{j}(t)$ as the solution evolves in time to again equidistribute its arc-length. The discrete solutions of the steady state of (1.1) using the adaptive mesh are steady states of this method. We show through some 
numerical experiments that the discrete solution with the transition layer placed at $x=1 / 2$ is unstable when $\varepsilon<\varepsilon_{S B}$ and that for a range of initial data, the adaptive solution converges to a problem with an asymmetrically placed transition layer. Thus the spurious solutions have a marked effect on the dynamics of the computed profile, with the metastability of the asymmetric states of the continuous problem being replaced by the stability of these states in the discrete problem.

The basic reason for this rather peculiar behavior is that in the continuous problems supersensitivity implies that the location of the transition layer is uniquely determined by exponentially small perturbations to the boundary conditions. For small $\varepsilon$ the effect of these perturbations is easily overcome by the errors introduced by the discretization process. An adaptive method which places mesh points in regions of high gradient only does not seem to affect this process. An interesting question is whether the correct location of the transition layer could be obtained by using a numerical method which places points close to the boundary, and we leave this for future investigation. We expect that the ideas in this paper are also relevent to problems which are only algebraically sensitive to the small diffusion parameter, but that then the effects will be less dramatic.

The remainder of this paper is organized as follows. In section 2 we derive a suitable numerical method for the steady state of (1.1) which incorporates a meshadaption procedure based upon a generalization of the well-known arc-length method. In section 3 we analyze this method when applied to the examples given. In particular, we determine the nature of the (discrete) solution branches as parametrized by $\varepsilon$ and estimate the location of bifurcation points where the nonspurious computed solution loses stability. We also study the form of the solution for $\varepsilon$ small. In section 4 we describe some numerical calculations of the steady state branches and compare them with the analytic estimates in section 3 . Finally, in section 5 we make some time-dependent calculations of the solutions of (1.1), based upon the moving-mesh strategy of [8], and show how the spurious steady state solutions affect the underlying dynamics of the problem.

2. Adaptive mesh discretizations of the steady BVP. Our derivation of discretization of the steady state of the equations is based upon the conservation form

$$
\begin{gathered}
\varepsilon u_{x}-F(u)=-k / 2, \\
u(0)=A, \quad u(1)=-A,
\end{gathered}
$$

where the constant $k$ is to be determined. In section 5 we will look at the timedependent formulation.

Let $U$ be the discretized solution variable $u$ and $H$ the vector of mesh spacings so that

$$
\begin{aligned}
& U=\left(u_{0}, u_{1}, \ldots, u_{J}\right)^{T}, \\
& H=\left(h_{1}, h_{2}, \ldots, h_{J}\right)^{T} .
\end{aligned}
$$

Thus the $h_{j}$ determine the (nonuniform) mesh distribution and $J$ is the number of grid cells. Hence $x_{l}=\sum_{j=1}^{l} h_{j}$ and $u_{j}$ denotes our approximations to $u\left(x_{j}\right)$. Discretizing the conservation equation over the interval $\left[x_{j}, x_{j+1}\right]$ and taking the mean of $F(u)$ over this interval we obtain

$$
\left\{\begin{array}{c}
\varepsilon \frac{u_{j+1}-u_{j}}{h_{j+1}}=\frac{1}{2}\left(F\left(u_{j+1}\right)+F\left(u_{j}\right)-k\right), \quad j=0, \ldots, J-1, \\
u_{0}=A, \quad u_{J}=-A .
\end{array}\right.
$$


Although we will mainly work with this discretization, we observe that subtracting two succesive such equations gives the quivalent formulation

$$
\varepsilon\left(\frac{u_{j+1}-u_{j}}{h_{j+1}}-\frac{u_{j}-u_{j-1}}{h_{j}}\right) / h=\frac{1}{2}\left(F\left(u_{j+1}\right)-F\left(u_{j-1}\right)\right) / h,
$$

where $h=\left(h_{j+1}+h_{j}\right) / 2$.

If the mesh is specified, $(2.2)$ represents $(J+2)$ equations for $(J+2)$ unknowns $U$ and $k$. Our interest, however, lies in the case where the mesh is adaptive so that the $J$ mesh parameters forming the vector $H$ are calculated as part of the solution. Thus we must specify further equations for the mesh. A common approach to mesh adaption is to use an equidistribution principle; see [8], [12], [13], [14], [20]. In these methods, there is a function $M\left(u, u_{x}, x\right)>0$, commonly called a monitor function, such that, if $x_{i}$ is the location of the $i$ th mesh point (with $x_{0}=0$ and $x_{J}=1$ ), then

$$
\int_{x_{i}}^{x_{i+1}} M\left(u, u_{x}, x\right) d x=\frac{1}{J} \int_{0}^{1} M\left(u, u_{x}, x\right) d x .
$$

In practice, of course, the integrals are approximated on the mesh.

Different monitor functions lead to different meshes with varying properties, and the "correct" choice of monitor function is not always clear. In [4] a monitor function for a certain two-point BVP is shown to give "optimal" accuracy in a suitable norm. Alternatively, it is proposed in [1] that for certain partial differential equations invariant under various rescaling groups, the most appropriate monitor function to give the correct dynamics of the solution is one which generates meshes that scale in a similar manner to the exact solution. These various considerations notwithstanding, the most commonly used monitor function is arc-length, for which

$$
M\left(u, u_{x}\right) \equiv \sqrt{1+u_{x}^{2}}
$$

see [20], [18]. Here we consider a monitor function which is a generalization of this one so that

$$
M\left(u, u_{x}\right) \equiv \sqrt{(1-\alpha)+\alpha u_{x}^{2}} .
$$

This formulation reduces to arc-length equidistribution if $\alpha=\frac{1}{2}$, gives a uniform mesh if $\alpha=0$, and gives equidistribution with respect to $\left|u_{x}\right|$ if $\alpha=1$. By varying $\alpha$ we may relate the properties of various mesh-adaptation principles, including the limiting case $\alpha=0$ of a uniform mesh.

To equidistribute the mesh we thus aim to choose $H$ such that

$$
\int_{x_{j-1}}^{x_{j}} \sqrt{(1-\alpha)+\alpha u_{x}^{2}} d x=\int_{x_{j}}^{x_{j+1}} \sqrt{(1-\alpha)+\alpha u_{x}^{2}} d x .
$$

Approximating the arc-length to be constant within the interval yields

$$
h_{j}^{2}\left((1-\alpha)+\alpha u_{x}^{2}\right)_{j-1 / 2}=h_{j+1}^{2}\left((1-\alpha)+\alpha u_{x}^{2}\right)_{j+1 / 2}, \quad j=1, \ldots, J-1 .
$$

A natural discretization of this equation gives

$$
\alpha\left(u_{j}-u_{j-1}\right)^{2}+(1-\alpha) h_{j}^{2}=\alpha\left(u_{j+1}-u_{j}\right)^{2}+(1-\alpha) h_{j+1}^{2}, j=1, \ldots, J-1 .
$$


Since (2.4) yields only $J-1$ equations as $j$ ranges from $1, \ldots, J-1$ we need the additional constraint that

$$
\sum_{j=1}^{J} h_{j}=1 .
$$

Thus (2.4), (2.5) give $J$ more equations for the unknowns $h_{j}$. Coupled with (2.2), this gives us a system of $2 J+2$ equations and unknowns. Since (2.4) is independent of $j$, the quantity on each side is a constant $s^{2}$. Thus,

$$
\left\{\begin{array}{c}
\alpha\left(u_{j}-u_{j-1}\right)^{2}+(1-\alpha) h_{j}^{2}=s^{2}, \quad j=1, \ldots, J, \\
\sum_{j=1}^{J} h_{j}=1 .
\end{array}\right.
$$

Thus (2.2), (2.6) form $2 J+3$ equations in the $2 J+3$ unknowns $\left\{u_{j}\right\}_{j=0}^{J}, k,\left\{h_{j}\right\}_{j=1}^{J}$, and $s$. This set of equations can be written as

$$
N(Q, \varepsilon, \alpha)=0
$$

where $Q=\left(U^{T}, k, H^{T}, s\right)^{T} \in \mathbb{R}^{3 J+3}$ and $N: \mathbb{R}^{2 J+3} \times \mathbb{R} \times \mathbb{R} \rightarrow \mathbb{R}^{2 J+3}$. By a solution we will always mean a real vector $Q \in \mathbb{R}^{2 J+3}$ with $h_{j}>0 \forall j=1, \ldots, J$.

In section 5 we generalize these ideas to include both the time-dependence of $u$ and a dynamic strategy for moving the mesh points.

3. An analysis of the discretized model equations. To analyze the solutions of (2.2), (2.6) considered as functions of $\varepsilon$ and $\alpha$ we apply techniques from bifurcation theory, determining the existence of a symmetric solution branch and then looking for asymmetric solutions bifurcating from it as $\varepsilon$ varies. We can then compare the qualitative properties of the discrete solution with those of the continuous solution. To start our investigation, we take $\varepsilon$ to be large and establish the existence of a unique, monotone decreasing, and symmetric solution of the discrete equations. This we refer to as the main solution branch and we establish some general properties of the symmetric and monotone decreasing solutions of (2.2), (2.6). As $\varepsilon$ is reduced, the main solution branch remains symmetric but loses monotonicity. However, further asymmetric solutions of (2.2), (2.6) arise. In particular, we show that there are symmetry breaking bifurcation points on the main branch leading to such asymmetric solutions. It is interesting that (as $\varepsilon$ is reduced) these occur before the main branch loses monotonicity. If $\alpha=1$, however, then the symmetry breaking points do not exist. Finally, we apply the implicit function theorem to show that, provided $\varepsilon$ is sufficiently small, then we may construct many solutions of the finite difference scheme, some of which may have arisen at the symmetry breaking points.

3.1. The solution for $\varepsilon \gg 1$. If we consider solutions which are bounded in the limit of $\varepsilon \rightarrow \infty$ so that $F\left(u_{j}\right)$ is bounded, and set $-k / 2=\varepsilon K$, then in this limit equation $(2.2)$ reduces to

$$
\frac{u_{j+1}-u_{j}}{h_{j+1}}=K
$$

Using the boundary conditions, $u_{0}=A$ and $u_{J}=-A$, this gives

$$
u_{j}=A+K \sum_{i=1}^{j} h_{i} .
$$


Therefore, to satisfy the last equation in (2.2) and (2.5), we require that $K=-2 A$. Thus, to leading order in $1 / \varepsilon$,

$$
u_{j}=A\left(1-2 \sum_{i=1}^{j} h_{i}\right) .
$$

To obtain the location of the mesh points we substitute these identities into the equation (2.6) to give

$$
4 \alpha\left(A^{2} h_{j}^{2}\right)+(1-\alpha) h_{j}^{2}=s^{2} .
$$

Thus $h_{j}$ is a constant which must therefore equal $1 / J$. Hence we have

$$
\begin{gathered}
h_{j}=\frac{1}{J}, \\
J^{2} s^{2}=4 \alpha A^{2}+(1-\alpha) .
\end{gathered}
$$

Hence, the unique bounded asymptotic solution of (2.2) for $\varepsilon \rightarrow \infty$ is given by

$$
\begin{gathered}
u_{j}=A\left(1-\frac{2 j}{J}\right), \\
h_{j}=\frac{1}{J}, \\
J^{2} s^{2}=4 \alpha A^{2}+(1-\alpha) .
\end{gathered}
$$

This is, in fact, the exact solution of the steady solutions of (1.1) at $\varepsilon=\infty$, sampled on a uniform grid.

3.2. Properties of the main solution branch. The solution constructed above for $\varepsilon \gg 1$ has a uniform mesh and is both monotonic, so that $u_{j}>u_{j+1}$, and symmetric, so that

$$
u_{j}=-u_{J-j} \quad \text { and } \quad h_{j}=h_{J+1-j} .
$$

Both the symmetry and monotonicity of the discrete solution are in qualitative agreement with the true solution of the continuous problem. As $F(u)$ satisfies the symmetry condition $F(u)=F(-u)$, then as $\varepsilon$ is reduced the equations (2.2), (2.6) will continue to admit a symmetric solution satisfying (3.2). For large enough $\varepsilon$ this solution will also be monotonic with $u_{j}>u_{j+1}$. As $\varepsilon$ is reduced then, in general, there will be a first value of $\varepsilon$ at which asymmetric solutions (not satisfying (3.2)) bifurcate from it and a further (smaller) value at which monotonicity is lost.

We now study how the monotone and symmetric solutions vary as $\varepsilon$ reduces. In particular, we examine the way in which the mesh changes from being uniform and obtain some bounds for the values of $h_{j}$; these are useful subsequently in finding bifurcation points. For this we assume that the function $F(u)$ satisfies the following conditions:

$$
\begin{gathered}
F(u)=F(-u), \quad F(0)=0, \\
d F / d u=G(u), \quad G(u)>0 \quad \text { if } \quad u>0, \quad G(u)>G(v) \quad \text { if } \quad u>v>0,
\end{gathered}
$$

where $G(u)$ is bounded for finite $u$. This includes Burgers's equation for which $f(u)=$ $u^{2} / 2$ and $G(u)=u$. 
Lemma 3.1. Let $\left\{\left\{u_{j}\right\}_{j=0}^{J}, k,\left\{h_{j}\right\}_{j=1}^{J}, s\right\}$ be a symmetric, monotone-decreasing solution of (2.2), (2.6) satisfying (3.2) with

$$
u_{j}>u_{j+1}
$$

Suppose further that $F(u)$ satisfies (3.3), (3.4) and $\alpha>0$. Then, for $j<J / 2$ we have

$$
h_{j}>h_{j+1}
$$

and

$$
\Delta_{j}:=u_{j}-u_{j+1}>\Delta_{j-1}
$$

COROllary 3.2. Under the same conditions as in Lemma 3.1 it follows that, for $J>2$,

$$
A\left(1-\frac{2}{J}\right)<u_{1}<A
$$

and

$$
\sqrt{4 A \alpha+(1-\alpha)}<J s<2 A \sqrt{\alpha}+\sqrt{1-\alpha}
$$

Proof of Lemma 3.1. It follows from (2.2) that for a monotone decreasing solution, the function

$$
a_{j}:=\frac{F\left(u_{j+1}\right)+F\left(u_{j}\right)}{2}-k
$$

is negative, and from (3.3) it is monotone decreasing if $j<J / 2$. Consequently, $a_{j}^{2}$ is monotone increasing if $j<J / 2$. Now combining (2.2), (2.6) we have

$$
h_{j+1}^{2}\left(\alpha \frac{a_{j}^{2}}{4 \varepsilon^{2}}+(1-\alpha)\right)=s^{2} .
$$

It follows immediately if $\alpha>0$ that, as $s$ is independent of $j$, then as $a_{j}^{2}$ is increasing, then $h_{j+1}$ is decreasing in $j$. We immediately deduce from (2.6) that $\left(u_{j+1}-u_{j}\right)^{2}$ is increasing in $j$. If $\alpha=0$ then as

$$
\varepsilon^{2} \Delta_{j}^{2}=h_{j+1}^{2} a_{j}^{2}
$$

and $h_{j}$ is constant, we deduce that as $a_{j}^{2}$ is increasing, then so is $\Delta_{j}$. This proves the lemma.

Proof of Corollary 3.2. Inequality (3.7) follows simply from the observations that $h_{j}$ is symmetric in $j$ and is decreasing for $j<J / 2$ and that $\sum_{1}^{J} h_{j}=1$. Inequality (3.8) is similar and uses the elementary identity that $\sum \Delta_{j}=2 A$. To obtain the estimate (3.9) we make use of the geometric result that the least arc-length of the 
solution occurs when the points $u_{j}$ lie on a straight line of uniform gradient joining the two end-points, which has length $\sqrt{4 A \alpha+(1-\alpha)}$. Similarly, the greatest arc-length occurs when the solution is two horizontal line-segments through $u=A$ and $u=-A$ joined by a vertical line-segment through $x \in(0,1)$. Such a curve has arc-length $2 A \sqrt{\alpha}+\sqrt{1-\alpha}$.

In general the solution does not stay monotonic as $\varepsilon$ is reduced. As a final result on the monotonic solutions we give an estimate for the value of $\varepsilon$ at which they lose monotonicity

LEMMA 3.3. If the symmetric solution first loses monotonicity at $\varepsilon=\varepsilon^{*}$, as $\varepsilon$ decreases, then at $\varepsilon=\varepsilon^{*}, \quad u_{1}=A$ and

$$
\varepsilon^{*}\left(u_{2}-A\right)=\frac{h_{2}}{2}\left(F\left(u_{2}\right)-F(A)\right) .
$$

Consequently, there exists a $u^{*} \in\left[u_{2}, A\right]$ with

$$
\varepsilon^{*}=\frac{h_{2} G\left(u^{*}\right)}{2} .
$$

Proof. The solution first loses monotonicity when $\Delta_{j}=0$ for some $j$. As $\Delta_{j}$ is monotone increasing by Lemma 3.1, this must occur first when $j=0$ so that $u_{1}=A$. It then follows from taking $j=0$ in $(2.2)$ that $k=2 F(A)$. The first result then follows by considering (2.2) when $j=1$ and the second by dividing both sides of the identity by $u_{2}-A$ and applying the mean value theorem.

3.3. Symmetry breaking bifurcations. We now show that (2.2), (2.6) has further asymmetric solutions which bifurcate from the symmetric branch described above. Hence the uniqueness and symmetry of the solution is lost as $\varepsilon$ reduces. In particular, we show that there are symmetry breaking points at which asymmetric solutions (not satisfying (3.2)) bifurcate from the symmetric branch at a nonzero value of $\varepsilon$. To establish this we investigate the linearization of (2.2), (2.6) about the symmetric solution and establish the existence of an asymmetric null eigenvector at a specific value of $\varepsilon_{S B}$. This is the condition for a point on the symmetric branch to be an infinitesimal symmetry breaking bifurcation. Such a point is actually a symmetry breaking bifurcation point provided that certain nondegeneracy conditions are satisfied. Generically this is so, and we observe (numerically) that additional spurious solutions do indeed bifurcate at these points. Furthermore, these spurious solutions appear to persist for all $\varepsilon \rightarrow 0$. Owing to the action of the symmetry group, all such bifurcations are necessarily pitchfork bifurcations and lead to two solution branches $u^{+}$and $u^{-}$in a neighborhood of $\varepsilon_{S B}$ such that

$$
u^{-}(x)=-u^{+}(1-x) .
$$

Numerical calculations demonstrate that $u^{-}$and $u^{+}$only exist for $\varepsilon<\varepsilon_{S B}$.

The value of $\varepsilon_{S B}$ is determined in the following theorem.

THEOREM 3.4. If $F(u)$ satisifies (3.3), (3.4) and $\alpha<1$, there is a symmetry breaking bifurcation on the symmetric solution branch of (2.2), (2.6) at a point $\varepsilon=$ $\varepsilon_{S B}^{(1)}$, where $\varepsilon_{S B}^{(1)}$ satisfies the equation

$$
h_{1} G\left(u_{1}\right)=2 \varepsilon_{S B}^{(1)}\left(1+\frac{\alpha}{1-\alpha} \frac{\left(u_{1}-A\right)^{2}}{h_{1}^{2}}\right) .
$$


Equivalently,

$$
\varepsilon_{S B}^{(1)}=(1-\alpha) \frac{h_{1}^{3} G\left(u_{1}\right)}{2 s^{2}} .
$$

Further infinitesimal symmetry breaking bifurcations from the main symmetric branch will occur for (smaller) values of $\varepsilon_{S B}^{(j)}$ which satisfy

$$
\varepsilon_{S B}^{(j)}=(1-\alpha) \frac{h_{j}^{3} G\left(u_{j}\right)}{2 s^{2}}, \quad j=2, \ldots, J-1 .
$$

Lemma 3.5. If $F(u)$ satisfies (3.3), (3.4) and $\alpha<1$ then there must be a value of $\varepsilon$ satisfying (3.11). Such a value satisfies

$$
\varepsilon_{S B}>\varepsilon^{*}
$$

where $\varepsilon^{*}$ is the greatest value of $\varepsilon$ at which the main solution branch loses monotonicity.

Corollary 3.6. Consider a solution of the discrete Burgers equation with $F(u)=u^{2} / 2, \quad G(u)=u$, and $A=1$.

(i) If $\alpha=0$ then

$$
\varepsilon_{S B}^{(1)}=\frac{h_{1} u_{1}}{2}
$$

Furthermore

$$
\frac{1}{2 J}\left(1-\frac{2}{J}\right)<\varepsilon_{S B}^{(1)}<\frac{1}{2 J}
$$

(ii) For all $\alpha \in[0,1), \varepsilon_{S B}^{(1)}$ satisfies the following inequalities:

$$
\frac{(1-\alpha)\left(1-\frac{2}{J}\right)}{2 J(2 \sqrt{\alpha}+\sqrt{1-\alpha})^{2}}<\varepsilon_{S B}^{(1)}<\frac{J^{2}(1-\alpha)}{16(1+3 \alpha)} .
$$

Note. The particular case $\alpha=0$ corresponds to a solution of (1.4) using a nonadaptive mesh, and (3.13) was first derived in [16] using a different method from the one we shall now give. The estimates (3.14), (3.15) are new. In section 5 we compare these bounds with numerically computed values of the symmetry breaking point.

COROLlaRY 3.7. For the resulting solution of the discrete Burgers equation, if

$$
\varepsilon<\frac{(1-\alpha)(1-2 / J)}{2 J(2 \sqrt{\alpha}+\sqrt{1-\alpha})^{2}},
$$

then an infinitesimal symmetry breaking bifurcation has occurred on the main branch for a value $\varepsilon_{S B}>\varepsilon$.

We can draw some useful conclusions from the previous lemmas. If we first consider a mesh without adaptivity, numerical evidence indicates that if $\varepsilon>1 / 2 J$ then the computed solution is nonspurious, and if $\varepsilon<(1-2 / J) / 2 J$ then spurious solutions exist in agreement with (3.14). Both of these estimates are reasonable. The natural length scale of the transition layer is $\mathcal{O}(\varepsilon)$, and to obtain an adequate resolution of the transition we must have a mesh such that $1 / J$ is of the same magnitude as $\varepsilon$. 
Thus spurious solutions are likely to exist if this is not the case, and we have to take a minimum value of $J$ to limit the effects of spuriosity. For the value of $\varepsilon$ in (3.16) it is likely that there are additional nonsymmetric solutions to the original equations. This result (perhaps rather surprisingly) indicates that using an adaptive mesh does not significantly improve on this result, and a comparable value of $J$ may have to be taken to avoid spuriosity. This is true even though the adaptive method may place many more points in the actual transition layer than the nonadaptive one. Note, however, that the case $\alpha=1$ eliminates such spurious bifurcations. This is examined further in section 3.5 .

Proof of Theorem 3.4. To prove the theorem we consider a linearization of (2.2), (2.6) about a symmetric solution. For the original system to have a bifurcation point it is necessary that the linearization should have a nonzero null-eigenvector $\delta$ with

$$
\delta=\left(w_{0}, w_{1}, \ldots, w_{J} ; g_{1}, \ldots, g_{J} ; t ; l\right)^{T},
$$

where $w_{j}$ corresponds to the linearization of $u_{j}, g_{j}$ to $h_{j}, t$ to $s$, and $l$ to $k$, respectively. A linearization of (2.2), (2.6) implies that these components satisfy the following system of linear equations:

$$
\begin{aligned}
& \left(h_{j+1} G\left(u_{j}\right)+2 \varepsilon\right) w_{j}+\left(h_{j+1} G\left(u_{j+1}\right)-2 \varepsilon\right) w_{j+1}+\left(F\left(u_{j}\right)+F\left(u_{j+1}\right)-k\right) g_{j+1}-h_{j+1} l \\
& \quad=0
\end{aligned}
$$

$j=0, \ldots, J-1$,

$$
\alpha\left(\left(u_{j}-u_{j+1}\right) w_{j}+\left(u_{j+1}-u_{j}\right) w_{j+1}\right)+(1-\alpha) h_{j+1} g_{j+1}-s t=0,
$$

$j=0, \ldots, J-1$,

$$
\sum_{1}^{J} g_{j}=0
$$

together with the further boundary condition that

$$
w_{0}=w_{J}=0 .
$$

As $u_{j}=u_{J-j}$ and $G\left(u_{j}\right)=-G\left(-u_{j}\right)$ it follows (after some manipulation) that if $l=t=0$ then (3.18)-(3.20) admits an antisymmetric solution which breaks the symmetry condition (3.2) and satisfies

$$
w_{j}=w_{J-j}, \quad g_{j}=-g_{J+1-j}, \quad l=t=0 .
$$

By [7] it follows that if such a solution is nonzero, then $\varepsilon_{S B}$ is an infinitesimal symmetry breaking bifurcation point, and asymmetric solutions of (2.2), (2.6) bifurcate from the main branch at this point. Thus we must show that there exists such an $\varepsilon$ where a solution of the form (3.21) is indeed nonzero.

Setting $l=t=0$ in (3.18), (3.18) and taking $j=0$ we have

$$
\left(h_{1} G\left(u_{1}\right)-2 \varepsilon\right) w_{1}+\left(F(A)+F\left(u_{1}\right)-k\right) g_{1}=0
$$

and

$$
\alpha\left(u_{1}-A\right) w_{1}+(1-\alpha) h_{1} g_{1}=0 .
$$


The reduced system (3.22), (3.23) has a nonzero solution for which $w_{1} \neq 0$ and $g_{1} \neq 0$ iff

$$
\operatorname{det}\left(\begin{array}{cc}
h_{1} G\left(u_{1}\right)-2 \varepsilon & F(A)+F\left(u_{1}\right)-k \\
\alpha\left(u_{1}-A\right) & (1-\alpha) h_{1}
\end{array}\right)=0
$$

Calculating the determinant in (3.24) and simplifying the resulting expression using the identity

$$
\varepsilon\left(u_{1}-1\right)=F(A)+F\left(u_{1}\right)-k
$$

gives the expression (3.10). On substituting the definition of $s$ we have the equivalent condition

$$
D_{1} \equiv \frac{(1-\alpha) G\left(u_{1}\right) h_{1}^{3}}{2 s^{2}}-\varepsilon_{S B}=0 .
$$

We can now construct the resulting null-eigenvector satisfying (3.21). In this case, the equation for $w_{2}$ and $h_{2}$ is given by

$$
\left(\begin{array}{cc}
h_{2} G\left(u_{2}\right)-2 \varepsilon & F\left(u_{1}\right)+F\left(u_{2}\right)-k \\
\alpha\left(u_{2}-u_{1}\right) & (1-\alpha) h_{2}
\end{array}\right)\left(\begin{array}{c}
w_{2} \\
g_{2}
\end{array}\right)=\left(\begin{array}{c}
-\left(h_{2} G\left(u_{1}\right)+2 \varepsilon\right) w_{1} \\
\alpha\left(u_{2}-u_{1}\right) w_{1}
\end{array}\right) .
$$

This problem will have a solution $\left(w_{2}, g_{2}\right)$ provided that the matrix in (3.26) has a nonzero determinant that is

$$
(1-\alpha) h_{2}\left(h_{2} G\left(u_{2}\right)-2 \varepsilon\right)-\alpha\left(u_{2}-u_{1}\right)\left(F\left(u_{1}\right)+F\left(u_{2}\right)-k\right) \neq 0
$$

which, on rearranging, is equivalent to the condition

$$
D_{2} \equiv \frac{(1-\alpha) G\left(u_{2}\right) h_{2}^{3}}{2 s^{2}}-\varepsilon_{S B} \neq 0 .
$$

Here we note that the equivalent term $D_{1}$ is identically zero at the bifurcation. We shall show presently that a bifurcation first occurs when $u_{i}$ is monotone decreasing and $h_{i}$ decreases monotonically if $i<J / 2$. Thus if $D_{1}=0$ it immediately follows that as $G(u)$ is monotone, then $D_{2} \neq 0$. Hence a solution $w_{2}, g_{2}$ of (3.26) exists. By a similar procedure we may calculate the subsequent terms $\left(w_{i}, h_{i}\right)$.

We note that if $D_{1} \neq 0$ but $D_{2}=0$, then a further bifurcation will occur with a null-eigenvector for which $w_{1}=g_{1}=0$ but $w_{2}, g_{2} \neq 0$. Thus a series of symmetrybreaking bifurcations occurs when each of the terms $D_{i}$ is equal to zero. This completes the proof.

We now show that such a value of $\varepsilon$ must exist.

Proof of Lemma 3.5. We look at the quantity

$$
D_{1} \equiv \frac{(1-\alpha) G\left(u_{1}\right) h_{1}^{3}}{2 s^{2}}-\varepsilon .
$$

By the estimates of the last section, $u_{1}, h_{1}, s$, and $1 / s$ are bounded above as $\varepsilon \rightarrow \infty$, and hence $D_{1}$ is negative for large $\varepsilon$. On the contrary, as $\varepsilon$ decreases, then if the solution remains monotone, these values are also bounded below (and are positive) so that $D_{1}$ is positive for small $\varepsilon$. Thus there must exist a value of $\varepsilon$ at which $D_{1}=0$. 
Now suppose that the main solution loses monotonicity when $\varepsilon=\varepsilon^{*}$. At such a point $U_{1}=A$ and hence from $(2.6)$ we have that $(1-\alpha) h_{1}^{2}=s^{2}$. Thus at this point

$$
D_{1}=\frac{h_{1} G(A)}{2}-\varepsilon^{*} .
$$

Now, from Lemma 3.3 we have

$$
\frac{h_{2} G\left(u^{*}\right)}{2}-\varepsilon^{*}=0
$$

where $u^{*}<A$. Thus, as $G(u)$ is monotone increasing in $u$ and $h_{2}<h_{1}$ we have that $D_{1}$ is positive if $\varepsilon=\varepsilon^{*}$. Thus $D_{1}$, which first becomes zero at a value $\varepsilon_{S B}>\varepsilon_{m}$ where the solution is still monotone.

Proof of Corollaries 3.6 and 3.7. The results in Corollaries 3.6 and 3.7 follow immediately from an application of the estimates of $u_{1}, h_{1}, s$ in Corollary 3.2 to the identity (3.11).

3.4. Asymptotic solutions when $\varepsilon \ll 1$. The symmetry breaking bifurcation generates solutions additional to the main branch for small values of $\varepsilon$. In fact, for very small values of $\varepsilon$ there are a very large number of solutions of (2.2), (2.6) As stated in the introduction, these are numerical perturbations of the many weak solutions which exist when $\varepsilon=0$, closely related to the supersensitivity of the underlying problem. To determine these we first recast (2.2) in the form

$$
\varepsilon\left(u_{j+1}-u_{j}\right)+\frac{1}{2} h_{j+1}\left(F\left(u_{j+1}\right)+F\left(u_{j}\right)-k\right)=0 .
$$

We show that the combined system (3.29), (2.6) has solutions when $\varepsilon=0$ and we use the implicit function theorem to deduce the existence of solutions when $\varepsilon>0$ as $\mathcal{O}(\varepsilon)$ perturbations of the $\varepsilon=0$ case.

Setting $\varepsilon=0$ in (3.29) gives

$$
h_{j+1}\left(F\left(u_{j+1}\right)+F\left(u_{j}\right)-k\right)=0,
$$

together with the mesh-defining equations (2.6) and the boundary condition

$$
u_{0}=A, \quad u_{J}=-A .
$$

We see immediately that this system has a solution if either

$$
h_{j+1}=0
$$

or

$$
F\left(u_{j+1}\right)+F\left(u_{j}\right)-k=0 .
$$

The values of $j$ for which $h_{j}=0$ correspond to points in the solution where there is a transition layer. We shall assume that the transition layer occurs for $L+1 \leq j \leq M$ over $J_{T} \equiv M-L$ intervals of the mesh. In the transition layer, it follows from (2.6) that if $h_{j}=0$ then a monotone decreasing solution satisfies

$$
u_{j+1}-u_{j}=-s / \sqrt{\alpha} .
$$




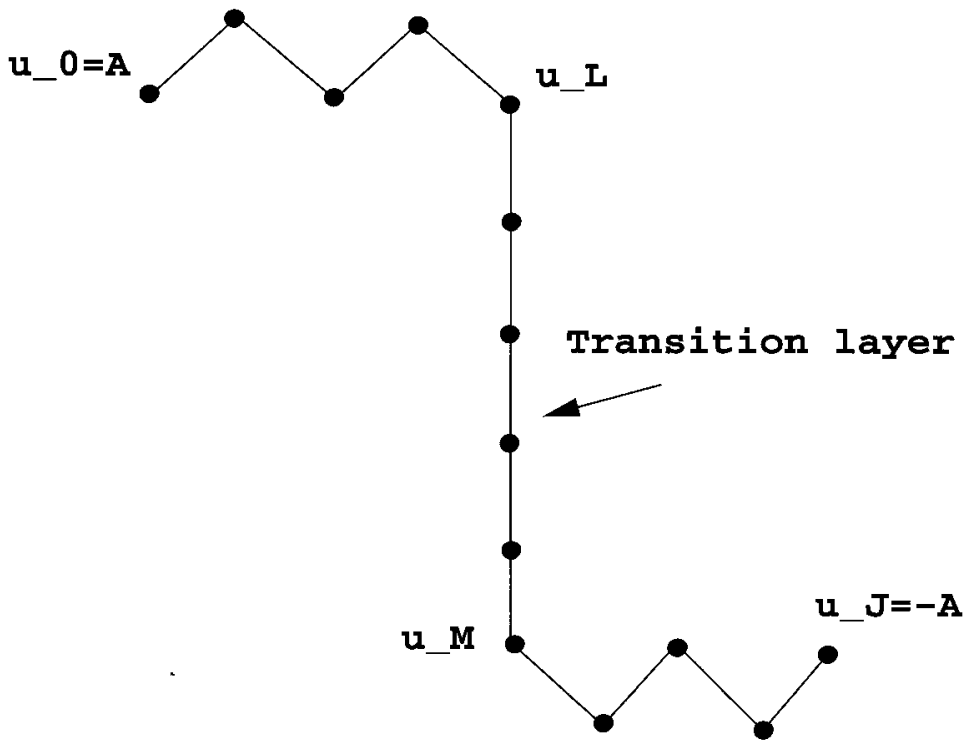

FIG. 1. The form of the solution when $\varepsilon=0$ showing the transition layer.

For values of $j$ for which $j$ and $j+2$ are not in the transition layer we have from applying (3.32) twice that

$$
F\left(u_{j}\right)=F\left(u_{j+2}\right),
$$

and hence from the monotonicity of $F(u)$ we deduce that

$$
u_{j}=u_{j+2} .
$$

Thus there is a constant $\delta$ for which

$$
\left(u_{j+1}-u_{j}\right)^{2}=\left(u_{j+2}-u_{j+1}\right)^{2} \equiv 4 \delta^{2} .
$$

A solution of this form is illustrated in Figure 1.

From this result and from (3.32) we may deduce that, since $u_{0}=A$,

$$
u_{1}=A+2 \delta, \quad u_{2}=A, \quad u_{J-1}=-A-2 \delta, \quad u_{J-2}=-A, \text { etc. }
$$

These results imply that $h_{j}$ is constant over all intervals outside the transition layer and takes the value

$$
h_{j} \equiv H=\frac{1}{J-J_{T}}
$$

so that

$$
4 \alpha \delta^{2}+(1-\alpha) H^{2}=s^{2} .
$$

By (3.33) we have

$$
u_{M}=u_{L}-J_{T} s / \sqrt{\alpha},
$$


and this gives another relationship between $s$ and $\alpha$ since $u_{M}$ and $u_{L}$ are determined by the parity of $L$ and $M$.

The solution of the system when $\varepsilon=0$ is thus characterized completely by the values of $H, \delta$, and $s$, each of which is given uniquely by the values of $L$ and $M$ (and hence of $J_{T}$ ). Indeed, the solution is independent of the function $F(u)$. We observe that if $L$ is even, then $u_{L}=A$, and if $L$ is odd, then $u_{L}=A+2 \delta$, with similar results for $u_{M}$. This gives us three cases of solutions to consider.

Case 1: $L$ even and $J-M$ even. In this case it follows from (3.35) that $u_{L}=A$, $u_{M}=-A$. Thus summing the expression (3.33) over the transition layer we deduce that

$$
s=2 A \sqrt{\alpha} / J_{T} .
$$

Substituting this expression into (3.37) gives the following expression for $\delta$ :

$$
\delta= \pm \sqrt{\frac{A^{2}}{J_{T}^{2}}-\frac{(1-\alpha)}{4 \alpha\left(J-J_{T}\right)^{2}}} .
$$

The expression (3.37) is valid provided that

$$
\Gamma_{1} \equiv \frac{\alpha}{1-\alpha}\left(J-J_{T}\right)^{2}-\frac{J_{T}^{2}}{4 A^{2}} \geq 0 .
$$

For example, when $\alpha=\frac{1}{2}, A=1$ this implies that

$$
J_{T} \leq \frac{2}{3} J
$$

We see that for this example the values of $\delta, s$, and $H$ are (up to a sign of $\delta$ ) given uniquely by the value of $J_{T}$ and do not depend upon the precise values of $L$ and $M$. Thus the solution derived can have a transition layer anywhere in the interval, provided that $L$ is even.

Case 2: $L$ odd and $J-M$ odd. We have $u_{L}=A+2 \delta, u_{M}=-A-2 \delta$ so that

$$
s=\left(\frac{2 A+4 \delta}{J_{T}}\right) \sqrt{\alpha} .
$$

Substituting into (3.37) implies that $\delta$ satisfies the quadratic equation

$$
\delta^{2}+\frac{1-\alpha}{4 \alpha\left(J-J_{T}\right)^{2}}=\frac{A^{2}(1+2 \delta)^{2}}{J_{T}^{2}} .
$$

In general, this equation has two solutions, and either may be observed. We will discuss these in section 4 .

Case 3: All combinations not included above. The analysis for this case follows similarly, and we have that $\delta$ satisfies the quadratic equation

$$
\delta^{2}+\frac{1-\alpha}{4 \alpha\left(J-J_{T}\right)^{2}}=\frac{A^{2}(1+\delta)^{2}}{J_{T}^{2}} .
$$

We now turn our attention to the perturbations of the above solutions when $\varepsilon$ is small. To do this we define the solution when $\varepsilon=0$ to be $U_{j}, H_{j}, S, K$, etc., so that $H_{j}=0$ in the transition layer. As $u_{j}$ is everywhere bounded, it is clear that 
the full problem described by (3.29), (2.6) is an $\mathcal{O}(\varepsilon)$ perturbation of that of (3.30), (3.30). Hence an application of the implicit function theorem implies that a solution of (3.29), (2.6) will take the general form

$$
\begin{gathered}
u_{j}=U_{j}+\mathcal{O}(\varepsilon), \quad s=S+\mathcal{O}(\varepsilon), \quad k=K+\mathcal{O}(\varepsilon), \\
h_{j}=H_{j}+\mathcal{O}(\varepsilon) \quad \text { outside the transition layer }
\end{gathered}
$$

and

$$
h_{j}=\mathcal{O}(\varepsilon) \quad \text { inside the transition layer. }
$$

We state the full result as follows.

THEOREM 3.8. Let $\alpha \in[0,1)$. In each of the cases $i=1,2,3$, the solution of (3.30), (2.6) perturbs smoothly in the manner defined by (3.44), (3.45), (3.46) provided that the corresponding variable $\Gamma_{i}$ defined in (3.41) for Case 1 (with similar expressions for the other two cases) satisfies the condition

$$
\Gamma_{i}>0
$$

Furthermore, if (3.47) is satisfied, then to leading order in $\varepsilon$, the mesh in the transition layer takes the form

$$
h_{j+1}=\frac{-2 \varepsilon s}{\left(F\left(u_{j+1}\right)+F\left(u_{j}\right)-k\right) \sqrt{\alpha}},
$$

where $u_{j}, s, k$ take the values calculated in the three cases above.

Proof. To prove that we can apply the implicit function theorem and deduce this result, we must show that the operator derived by linearizing (3.30), (2.6) about the solution $\left(U_{j}, H_{j}, S, K\right)$ is invertible or, equivalently, that it has no (nonzero) nulleigenvector. The proof of this is straightforward but technical, and details of this calculation are given in [2]

3.5. Existence and uniqueness of solutions for $\boldsymbol{\alpha}=1$. When $\alpha=1$ we have from (2.6) that $\left|u_{j}-u_{j+1}\right|=s$. Consequently, if the solution is monotone, we have that the value of $\Delta_{j}=u_{j}-u_{j+1}$ given in Lemma 3.1 is constant. As the solution can only lose monotonicity when $\Delta_{1}=0$, we deduce that a loss of monotonicity can only occur if $\Delta_{j}=0$ for all $j$. Thus $u_{j}$ is constant for all $j$, violating the boundary conditions. We deduce that the main solution branch remains monotone for all $\varepsilon$. As $\varepsilon$ tends to zero the only possible monotone solution of (3.30) is one for which $h_{1}=h_{J}=1 / 2, h_{j}=0$ otherwise, and there is a transition layer with $L=1$ and $M=J-1$. Similarly, from (3.25) we see that a symmetry breaking bifurcation can only occur when $\alpha=1$ if $\varepsilon_{S B}=0$. Thus, although the analysis in the previous section does not rule out the existence of nonmonotone spurious asymmetric solutions when $\alpha=1$ for small values of $\varepsilon$, we can deduce that such solutions cannot bifurcate from the main branch. More significantly, they do not alter the stability of the main branch. Thus taking $\alpha=1$ reduces the impact of the spurious solutions. We shall see numerical evidence for this in the next section.

4. Numerical results for the examples. This section presents the numerical solution of $(2.2)$, (2.6) obtained through continuation in both $\varepsilon$ and $\alpha$ using the package AUTO [5]. In all cases $J$ was kept fixed at $J=21$, and we take $F(u)=u^{2} / 2$ (Burgers's equation) and $A=1$. 


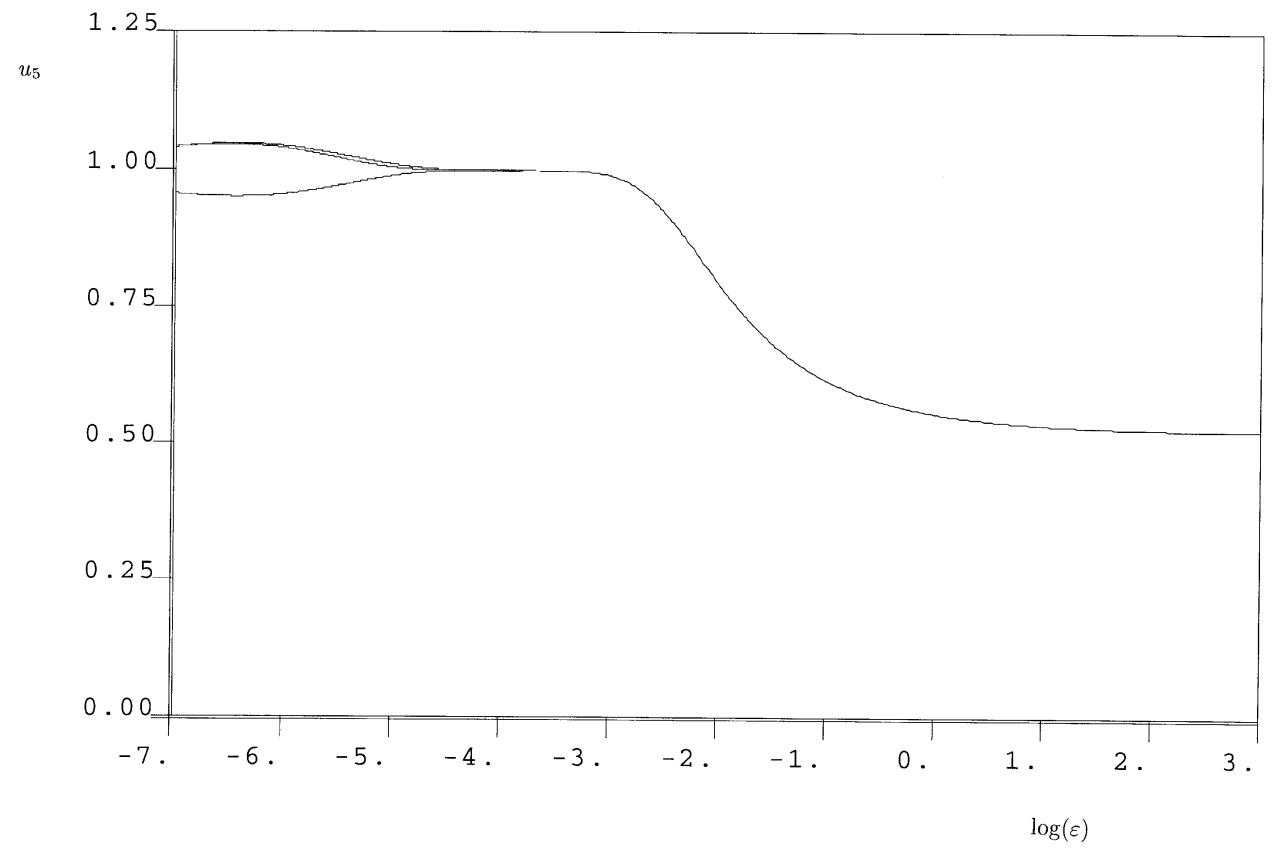

FIG. 2. The value of $u_{5}$ as a function of $\log (\varepsilon)$ when $\alpha=0$.

To compute a solution branch parameterized by $\varepsilon$ we take as a starting solution the monotone symmetric solution computed in section 3.1 with $\varepsilon=10$. The value of $\varepsilon$ is then reduced and all bifurcations and bifurcating branches computed. At the fixed value of $\varepsilon=1 \times 10^{-3}$ we also look at the solutions as functions of $\alpha$. Further, disconnected branches can be found for very small values of $\varepsilon$ by using the asymptotic solution (when $\varepsilon=0$ ) as a starting solution when $\varepsilon$ is small.

4.1. Continuation in $\varepsilon$. We take $\alpha$ to be fixed at the two values of $0,1 / 2$ and compute the bifurcation diagrams in the two cases. As a suitable measure of the solution we take the value of $u_{5}$, as this is effective in distinguishing between solutions with different locations of the transition layer.

Figures 2 and 3, then, show the corresponding bifurcation diagrams of the solution of (2.2), (2.6). Interestingly, in the second of these two figures, the bifurcating branches are much easier to distinguish as the point $u_{5}$ is moved into the transition layer. In Figure 4 we also present the value of $h_{1}$ as a function of $\varepsilon$ in the case of $\alpha=1 / 2$. In this figure we see that $h_{1}$ tends to one of the three limits of $1 / 7,1 / 8$, and 0 as $\varepsilon$ tends to zero.

It is clear from Figures 2 and 3 that when $\alpha=0$ and $\alpha=1 / 2$ there are symmetry breaking bifurcations from the main branch, as predicted by Theorem 3.4, leading to solution branches which are asymmetric. It is also clear that these solutions tend toward an asymptotic limit as $\varepsilon$ tends to zero. Using the notation of section 3.4 we may thus characterize these solutions by the values of $M$ and $L$ such that a transition layer occurs for $j$ between $L+1$ and $M$. These values are indicated on Figure 2. It is interesting to note that the solutions which have the transition layer at $x=0(L=0)$ as $\varepsilon \rightarrow 0$ and at $x=1(L=7)$ are the continuations of the two solutions $u^{-}, u^{+}$ which bifurcate from the main solution branch at the greatest value of $\varepsilon$. Subsequent 


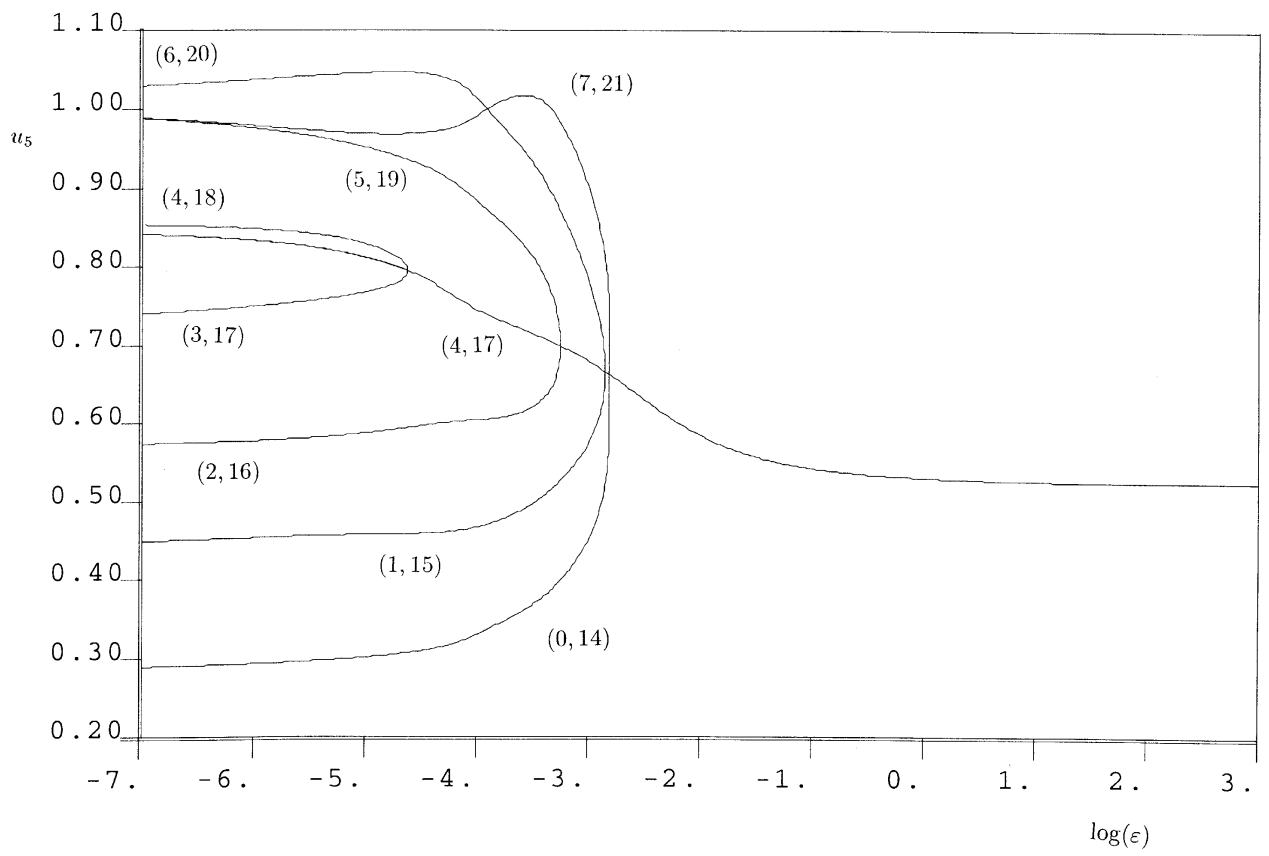

FIG. 3. The value of $u_{5}$ as a function of $\log (\varepsilon)$ when $\alpha=1 / 2$.

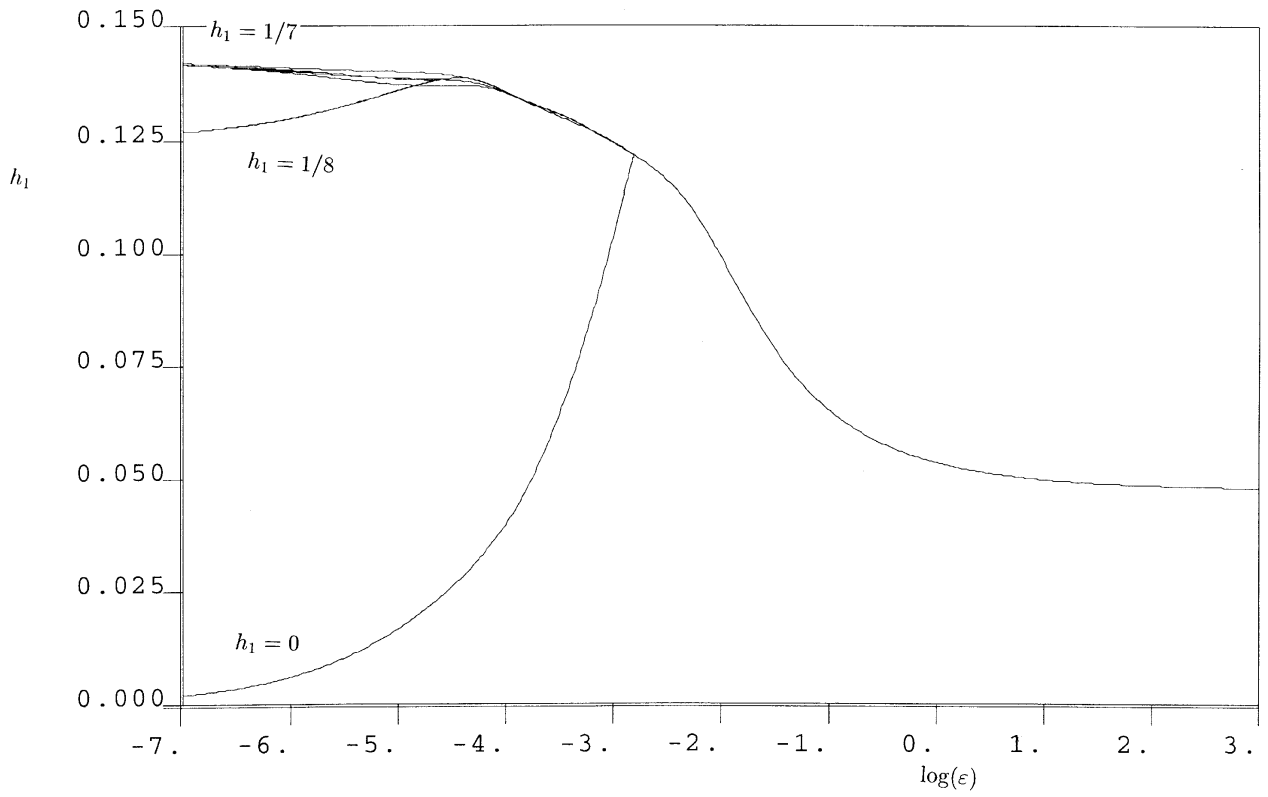

FIG. 4. The value of $h_{1}$ as a function of $\log (\varepsilon)$ when $\alpha=1 / 2$. 
solution branches which have transition layers closer to $x=1 / 2$ bifurcate from the main branch at smaller values of $\varepsilon$. In Figures 5 and 6 we present the complete set of solutions for $\varepsilon=1 \times 10^{-3}$ with $\alpha=0,1 / 2$, respectively. These clearly show the oscillatory structure predicted in section 3.4.

If $\alpha=0$ then the first bifurcation occurs when $\varepsilon=2.38 \times 10^{-2}$. If $\alpha=1 / 2$, bifurcations occur when $\varepsilon=6.081 \times 10^{-2}, 5.835 \times 10^{-2}, 3.896 \times 10^{-2}$, and $9.869 \times 10^{-3}$. Thus the value of $\varepsilon$ for the first of these bifurcation points actually increases when the adaptive procedure is used. If $\alpha=1 / 2$ then the location of the first of these bifurcation points can be estimated from (3.14), which predicts that $0.004787<\varepsilon_{S B}^{(1)}<2.45$. This is consistent with the above computations. In Figure 7 we give a graph comparing the estimates of (3.14) with the actual location of the bifurcation point. It is interesting to note from this graph that $\varepsilon_{S B}$ has a maximum value at about $\alpha=0.9$ before decreasing to zero as $\alpha$ tends towards 1 .

If we now take $\alpha=1$ then $u_{5}$ is fixed for all $\varepsilon$. However, the value of $h_{1}$ increases towards $1 / 2$ as $\varepsilon$ tends towards zero. This behavior can be seen in Figure 8, which plots $h_{1}$ as a function of $\varepsilon$ in this case. As predicted in section 3.5 we see that there are no bifurcations from the main solution branch. The resulting profile when $\varepsilon=1 \times 10^{-3}$ is given in Figure 9. From this figure we can see that the transition layer occurs at the right location but the grid points are concentrated in a small region.

4.2. Continuation in $\boldsymbol{\alpha}$. For continuation in the parameter $\alpha$ we have taken one of the solutions at $\alpha=\frac{1}{2}$ and $\varepsilon=10^{-3}$ from Figure 3 as a starting solution. The bifurcation diagram with $\alpha$ as the parameter is shown in Figure 10, for which $u_{2}$ is a convenient value to plot. This again shows a complex form with many solutions, although the structure of the bifurcations in this case is less clear than the continuation with $\varepsilon$.

4.3. Asymptotic properties of the solutions. As remarked above, it is clear that as $\varepsilon$ tends to zero, the computed solutions are converging to the asymptotic solution predicted in section 3.4. We now make this comparison more precise. The solutions at $\varepsilon=4 \times 10^{-5}$ from two different branches of the bifurcation diagram are shown in Figure 11. In the figure, Solution 1 has $L=4, M=17$, and $J_{T}=13$ and represents the symmetric solution on the main branch. In contrast, Solution 2 has $L=7, M=21$, and $J_{T}=14$ and has a maximal degree of asymmetry. Since $J=21$ for both cases, Solution 1 corresponds to Case 1 and Solution 2 corresponds to Case 3 , described in section 3.4.

To verify the asymptotic analysis for Solution 1 , we determine $\eta \equiv \frac{u_{1}-u_{0}}{2}$ from the numerical computations and compare it with the value of $\delta$ given by (3.40). This analysis implies that if $\varepsilon=0$ then

$$
\delta=4.4843 \times 10^{-2}, \quad h=1 / 8,
$$

so that in general $\eta=\delta+\mathcal{O}(\varepsilon)$. At $\varepsilon=7.9 \times 10^{-4}, \eta \approx 4.3 \times 10^{-2}$ and $|\eta-\delta| \approx$ $1.8 \times 10^{-3}$, and at $\varepsilon=4 \times 10^{-5}, \eta \approx 4.45 \times 10^{-2}$ and $|\eta-\delta| \approx 8.8 \times 10^{-5}$. These values show that $\delta-\eta$ scales as $\varepsilon$, which is consistent with the asymptotic value.

Solution 2 is an example of Case 3 of section 3.4. For this solution the equation (3.43) yields two values for $\delta$, namely,

$$
\delta=2 / 195 \quad \text { and } \quad \delta=0, \quad h=1 / 7 .
$$

Clearly, from the figure it is the latter value which is seen as a solution bifurcating from the main branch. Presumably the other solution appears at a fold bifurcation. 


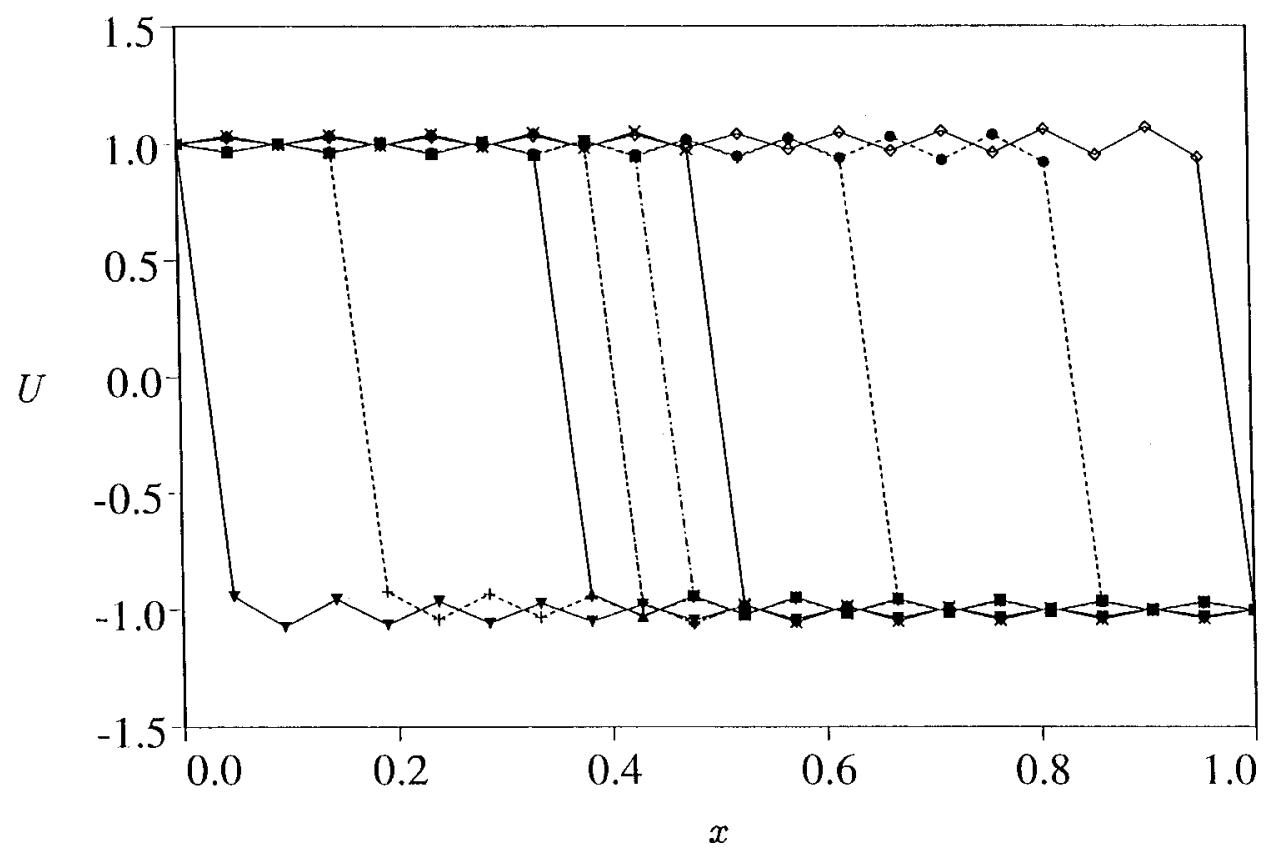

FIG. 5. The set of solutions when $\alpha=0$ and $\varepsilon=1 \times 10^{-3}$.

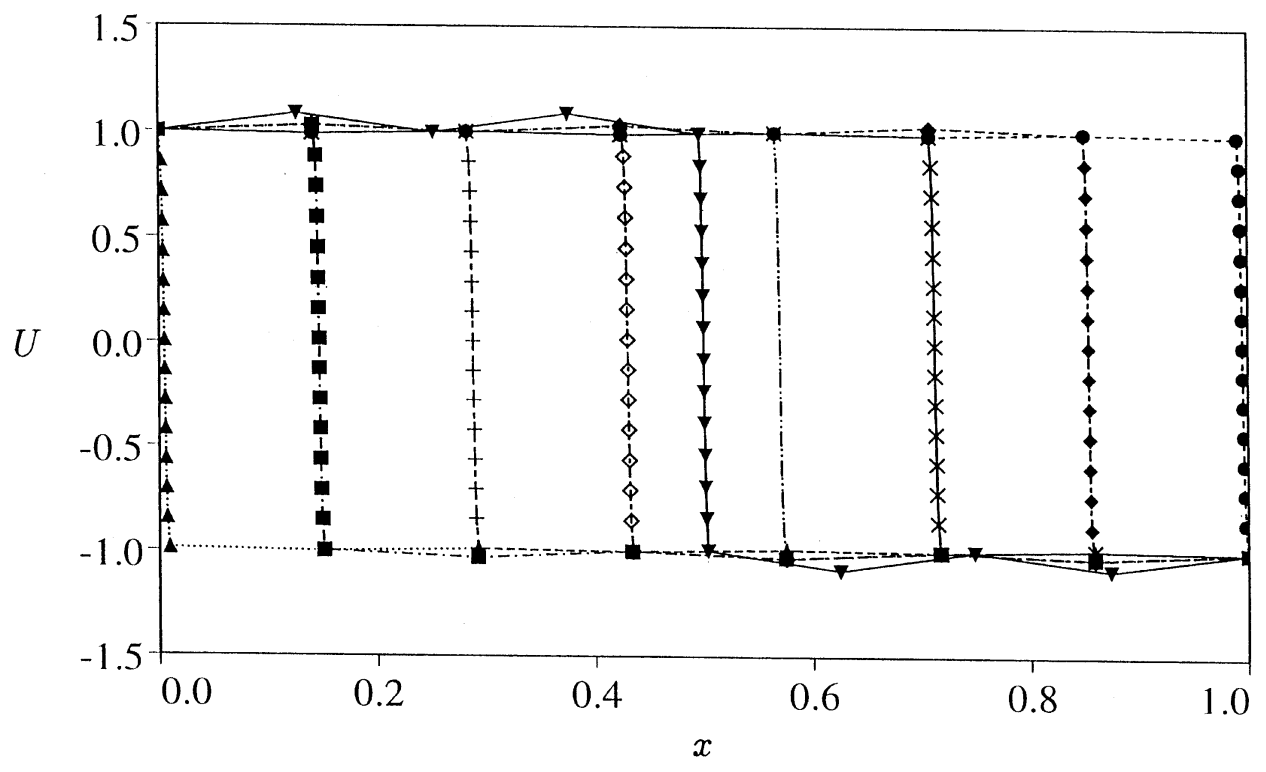

FIG. 6. The set of solutions when $\alpha=1 / 2$ and $\varepsilon=1 \times 10^{-3}$. 


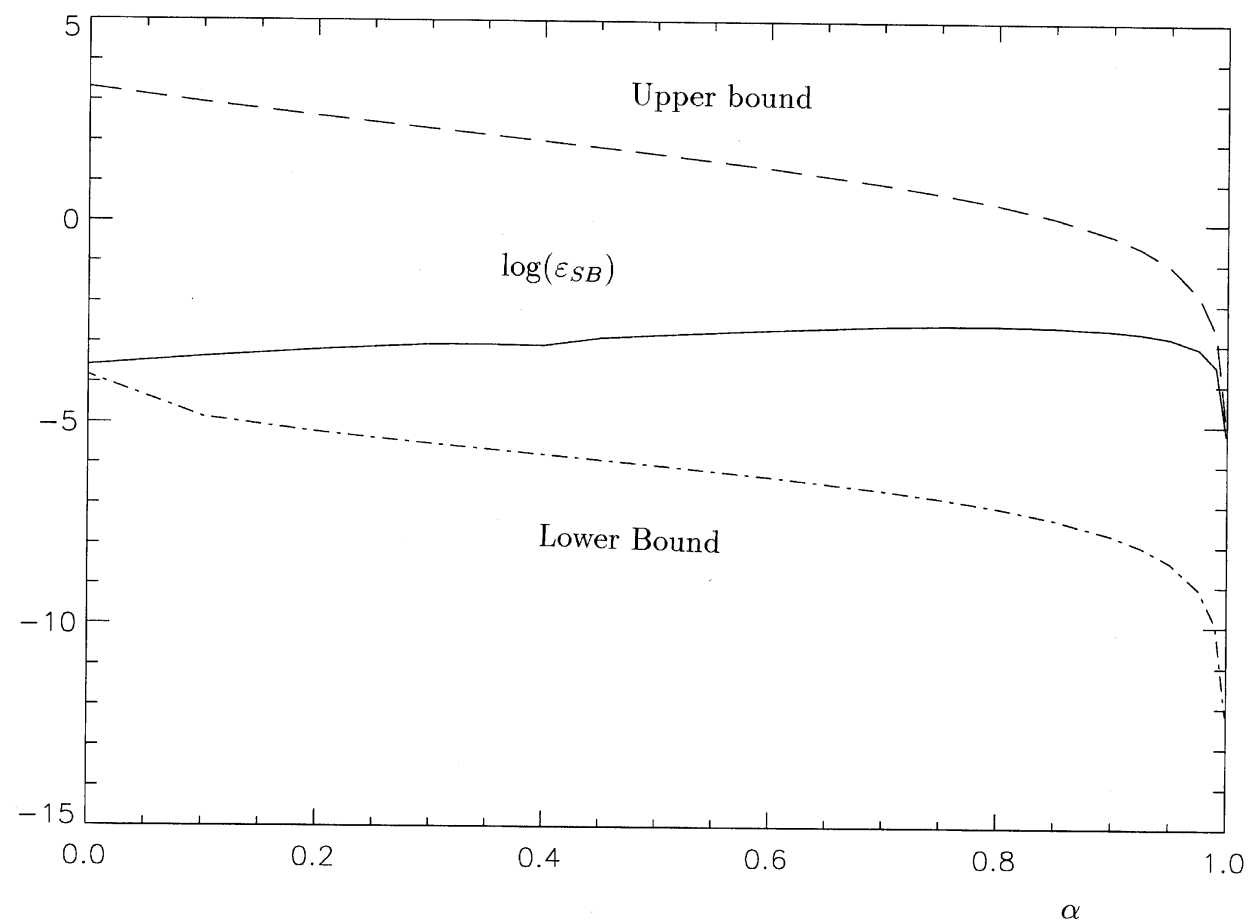

FIG. 7. A comparison of the calculated value of $\varepsilon_{S B}$ as a function of $\alpha$ with the upper and lower bounds given in Corollary 3.6.

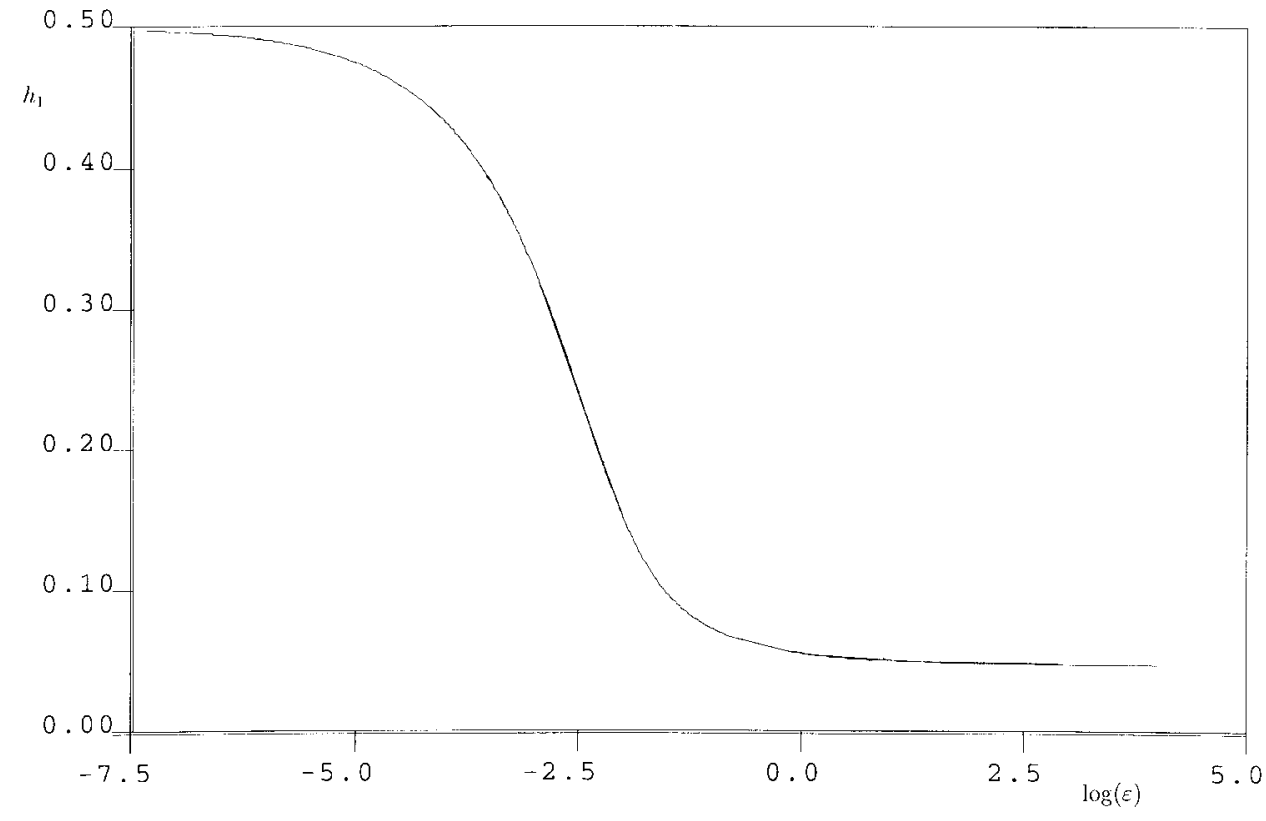

FIG. 8. The value of $h_{1}$ as a function of $\log (\varepsilon)$ when $\alpha=1$. 


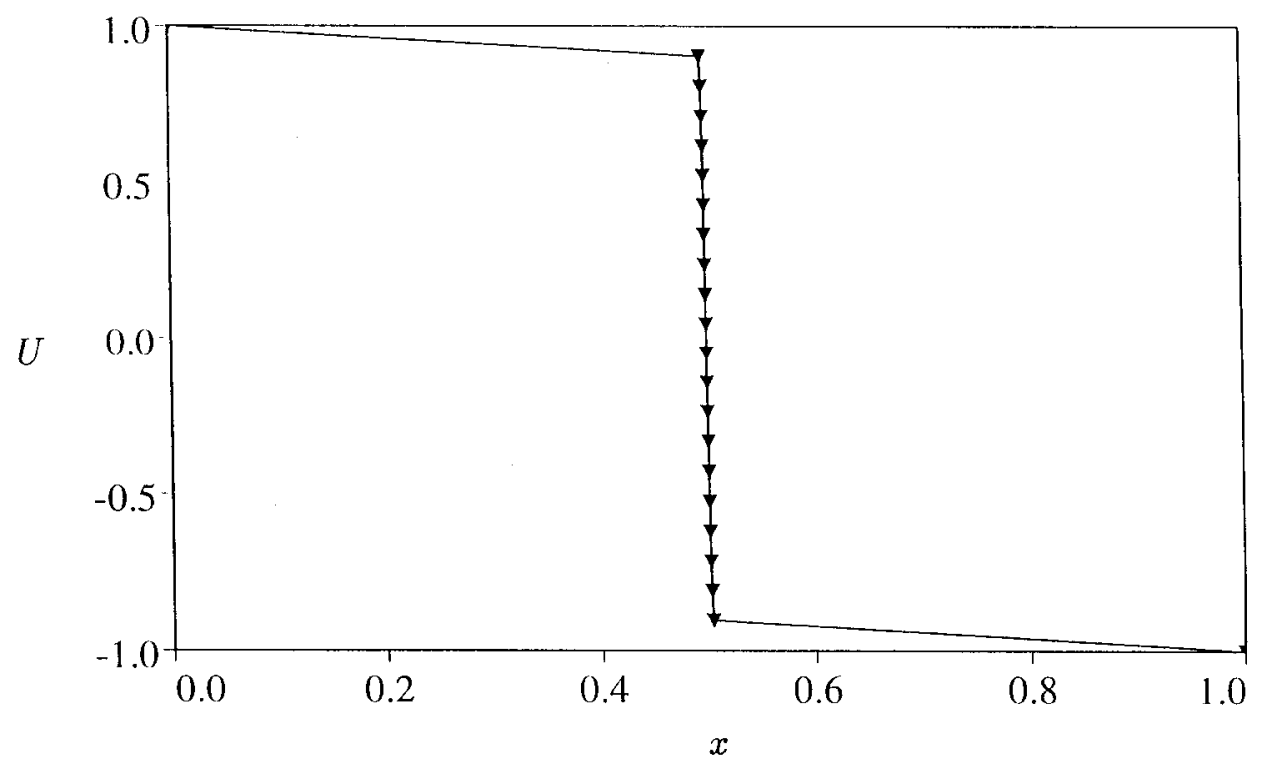

FIG. 9. The solution when $\alpha=1$ and $\varepsilon=1 \times 10^{-3}$.

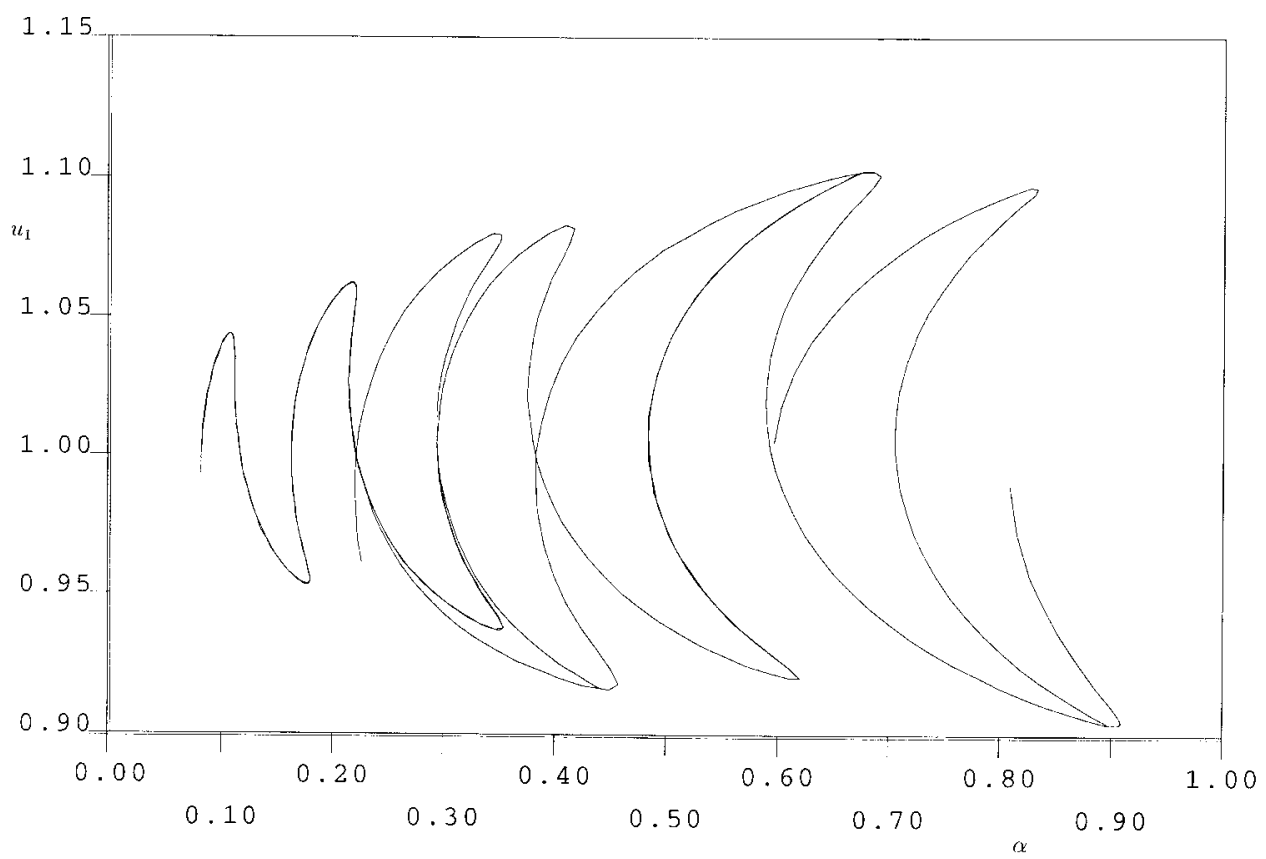

FIG. 10. The value of $u_{1}$ as a function of $\alpha$ when $\varepsilon=1 \times 10^{-3}$. 


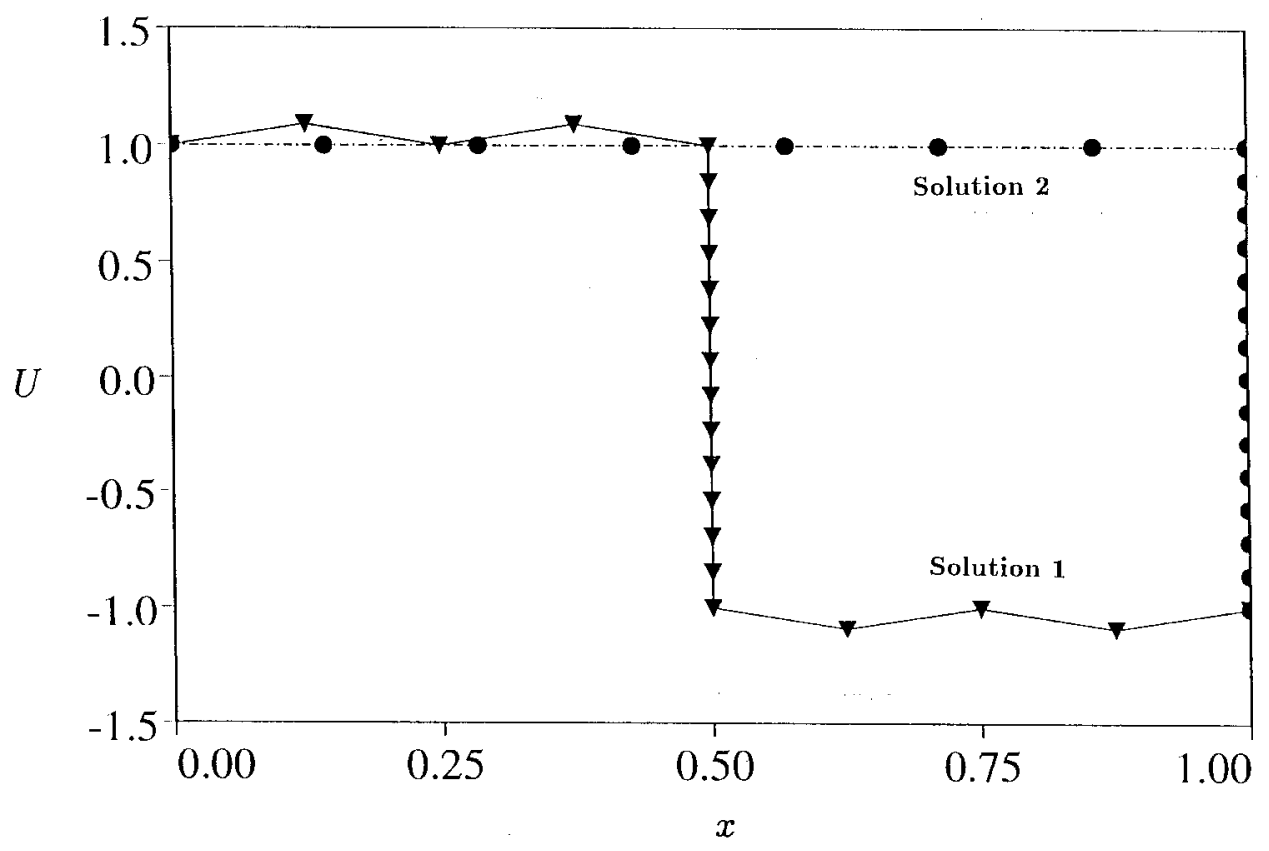

FIG. 11. Two of the solutions obtained when $\alpha=1 / 2$ and $\varepsilon=1 \times 10^{-3}$ showing a symmetric solution with a transition layer at $x=1 / 2$ and an asymmetric solution with a transition layer at $x=1$.

5. Time-like iterations to steady state. We now consider the implications of our steady state analysis for the solution of the full-time dependent problem (1.1), in particular determining which of the solutions constructed is stable. To do this we augment the partial differential equation with additional equations allowing the mesh points to evolve and for which (2.6) represents a steady state. Thus we can study the dynamical effect of the steady solution computed in section 3 . We consider meshes which evolve to equidistribute the quantity $M(u, x)$, and for simplicity in this section we only consider discretizations of Burgers's equation with $A=1$.

To apply such a moving mesh technique we follow [8] and introduce a new computational coordinate $\xi \in[0,1]$ and a mesh function $x(\xi, t)$ so that the $i$ th mesh point is at $x(i / J, t)$. We then introduce a second partial differential equation to describe the evolution of $x$. As the value of $x$ is now a function of time, we must recast Burgers's equation in a moving coordinate system, $x=x(\xi, t)$, so that

$$
\left\{\begin{array}{c}
u_{t}=\varepsilon u_{x x}-\frac{1}{2}\left(u^{2}\right)_{x}+u_{x} x_{t}, \\
u(0, t)=A, \quad u(1, t)=-A, \quad u(x, 0)=g(x) .
\end{array}\right.
$$

To evolve the mesh we then couple this with the following moving mesh equation given in [8]:

$$
\left\{\begin{array}{c}
\left(x_{t}\right)_{\xi \xi}=-\frac{1}{\tau}\left(M x_{\xi}\right)_{\xi}, \\
x(0, t)=0, \quad x(1, t)=1, \quad x(\xi, 0)=y(\xi),
\end{array}\right.
$$

where $0<\tau \ll 1$ is a relaxation parameter, $M(x(\xi, t), t)$ is the quantity to be equidistributed, and $y(\xi)$ determines the initial mesh distribution. In this section we take 
$\alpha=1 / 2$ and consider the arc-length monitor function $M=\sqrt{1+u_{x}^{2}}$. From (5.2) it can be seen that the solution equidistributes the quantity $M$ if it reaches steady state. The time evolution is introduced as a fast relaxation to equidistribution, avoiding the solution of differential algebraic equations for the evolution problem. Our purpose in this section is to study the stability and basins of attraction of equilibria under this natural evolution process. For the stability properties of the equation (5.2), see [13]. Other time-like iteration schemes for the moving mesh equations are studied in [17].

5.1. Discretization of the equation. Following [8], equations (5.1) and (5.2) are discretized using the method of lines. The spatial derivatives are, as before, approximated using central differences and the resulting stiff system of ODEs, for the solution variables and mesh locations, is solved using a stiff solver.

We set

$$
x_{j}(t)=\sum_{l=1}^{j} h_{l}(t)
$$

and define

$$
\begin{aligned}
& U(t)=\left(u_{0}(t), u_{1}(t), \ldots, u_{J}(t)\right)^{T}, \\
& X(t)=\left(x_{1}(t), x_{2}(t), \ldots, x_{J}(t)\right)^{T} .
\end{aligned}
$$

The equation (5.1) is discretized on a nonuniform mesh in $x$ to obtain

$$
\frac{d u_{j}}{d t}=P_{j}(U, X)+Q_{j}(U, X) \frac{d x_{j}}{d t}, \quad j=1, \ldots, J-1 .
$$

Here, the operator $P$ is as given in (2.3) so that

$$
P_{j}(U, X)=\frac{2 \varepsilon}{x_{j+1}-x_{j-1}}\left[\frac{u_{j+1}-u_{j}}{x_{j+1}-x_{j}}-\frac{u_{j}-u_{j-1}}{x_{j}-x_{j-1}}\right]-\frac{1}{x_{j+1}-x_{j-1}}\left[\frac{u_{j+1}^{2}}{2}-\frac{u_{j-1}^{2}}{2}\right],
$$

and we take a central difference discretization for the convective operator to give

$$
Q_{j}(U, X)=\frac{u_{j+1}-u_{j-1}}{x_{j+1}-x_{j-1}} .
$$

The equations (5.2) are then discretized on a uniform mesh in $\xi$, to give $\left(x_{t}\right)_{j+1}-2\left(x_{t}\right)_{j}+\left(x_{t}\right)_{j-1}=M_{j+\frac{1}{2}}\left(x_{j+1}-x_{j}\right)-M_{j-\frac{1}{2}}\left(x_{j}-x_{j-1}\right), \quad j=1, \ldots, J-1$, where

$$
M_{j+\frac{1}{2}}=\sqrt{1+\left(\frac{u_{j+1}-u_{j}}{x_{j+1}-x_{j}}\right)^{2}}
$$

This gives

$$
\frac{d X}{d t}=A R(U, X),
$$

where $A$ is the inverse of a tridiagonal matrix with diagonal elements -2 and offdiagonal elements 1 , and $R$ is a $(J-1)$ vector with $j$ th element

$$
R_{j}=M_{j+\frac{1}{2}}\left(x_{j+1}-x_{j}\right)-M_{j-\frac{1}{2}}\left(x_{j}-x_{j-1}\right) .
$$


This can also be written as

$$
\frac{d x_{j}}{d t}=\sum_{k=1}^{J-1} A_{j k} R_{k}, \quad j=1, \ldots, J-1 .
$$

Using this in (5.4) we get

$$
\frac{d u_{j}}{d t}=P_{j}(U, X)+Q_{j}(U, X) \sum_{k=1}^{J-1} A_{j k} R_{k}(U, X), \quad j=1, \ldots, J-1 .
$$

Then the equations (5.6) and (5.7) represent a system of ODEs,

$$
\frac{d Z}{d t}=G(Z)
$$

where $Z(t)=\left(u_{1}(t), \ldots, u_{J-1}(t), x_{1}(t), \ldots, x_{J-1}(t)\right)^{T}$. Therefore, (5.8) represents the system for the evolution in time of $u$ and $x$ at uniform grid locations in $\xi$. At steady state, these equations reduce to the mesh adaptation introduced earlier, in the case $\alpha=\frac{1}{2}$.

5.2. Numerical results of the unsteady computations. The solution branches in the bifurcation diagram of Figure 2 represent the equilibrium points of the system (5.8). Thus the stability of these solution branches can be analyzed using a linearized stability analysis of the system (5.8). This analysis can be performed by AUTO and the resulting stability properties of the branches when $A=1$ are given in Figure 12, where the solid lines represent the stable branches and the broken lines represent the unstable ones. The main branch undergoes supercritical and subcritical bifurcations alternately at the sucessive symmetry breaking bifurcation points $\varepsilon_{i}$. In particular, it loses stability as $\varepsilon$ is reduced below $\varepsilon_{1}=6.081 \times 10^{-2}$. Thus the effect of the spurious solutions is to destabilize the main symmetric solution branch. The secondary branch bifurcating from the third bifurcation point at $\varepsilon_{3}=3.896 \times 10^{-2}$ loses stability through a Hopf bifurcation at $\varepsilon_{H}=9.7 \times 10^{-3}$. Since the underlying partial differential equation cannot undergo a Hopf bifurcation, the oscillations resulting from the numerically observed Hopf bifurcation arise directly from the adaptive mesh strategy.

To study the stability characteristics of the branches under large perturbations we now consider evolution of the system (5.8) from specific initial data using the backward Euler method. We have computed the solution at $\varepsilon=1.0 \times 10^{-2}$. For this value of $\varepsilon$ there are seven equilibrium solutions, one on the main branch and two on each of the secondary branches bifurcating from $\varepsilon_{1}, \varepsilon_{2}$, and $\varepsilon_{3}$. For this value of $\varepsilon$, the main branch and the secondary branch bifurcating from $\varepsilon_{2}$ are unstable, whereas the secondary branches emanating from bifurcations at $\varepsilon_{1}$ and $\varepsilon_{3}$ are stable. Numerical experiments strongly imply that the stable solutions described above are the only attractors of the time-dependent problem, with each having a nonzero basin of attraction. It is thus of interest to ask which initial data is attracted to which branch. To gain insight into this question we chose as initial data for the unsteady computation, small perturbations of both the solution $U_{i}$ and the equidistributed mesh computed at the unstable equilibrium solutions. As a measure of the evolution of the system we define

$$
M_{a v}=\frac{1}{J} \sum_{j=0}^{J-1} M_{j+\frac{1}{2}}
$$




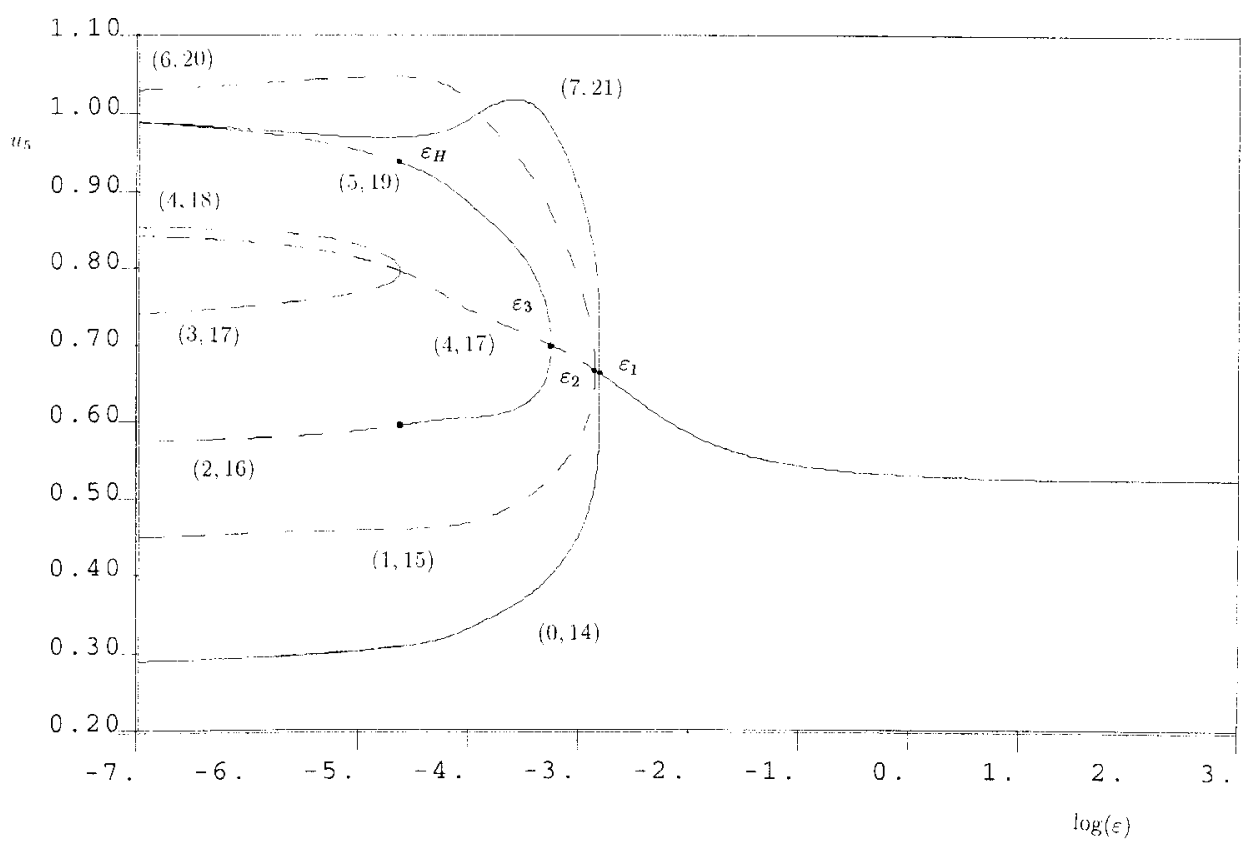

FIG. 12. The stability of the solutions described in Figure 3.

and

$$
\left\|M-M_{a v}\right\|=\sqrt{\sum_{j=0}^{J-1}\left(M_{j+\frac{1}{2}}-M_{a v}\right)^{2}},
$$

where $M_{j+\frac{1}{2}}$ is defined as in (5.5). Thus $\left\|M-M_{a v}\right\|$ represents some measure of the deviation of the solution from equidistribution. For all our computations we find that $\left\|M-M_{a v}\right\|$ increases initially as the solution moves away from the unstable branch. However, once the solution is close to a stable branch the deviation from equidistribution decays exponentially. The convergence history of the unsteady computation with starting solutions close to the unstable branches at $\varepsilon=1.0 \times 10^{-2}$ is shown in Figure 13. To obtain the results summarized in Curve 1, initial data is taken which is a perturbation of a symmetric solution labeled $(4,17)$ on the main branch. To obtain the results in Curves 2 and 3, two nearby sets of initial data are chosen which are both perturbations of the asymmetric unstable solution branch labeled $(6,20)$ bifurcating from the main branch at $\varepsilon_{2}$ but which lead to different time histories. In these time histories the solutions represented by Curves 1 and 2 both converge slowly toward the stable secondary branch emanating from $\varepsilon_{3}$ labeled $(5,19)$. The oscillations in these solutions, and the slow convergence, is due to the existence of complex eigenvalues of the linearized system which have real part close to zero. This follows from the fact that $\varepsilon$ is close to the Hopf bifurcation point at $\varepsilon_{H}$. In contrast, Curve 3 exhibits rapid convergence to the secondary branch emanating from $\varepsilon_{1}$ labeled $(7,21)$. Thus the secondary curve bifurcating from $\varepsilon_{2}$ in some sense divides the initial data which evolves toward the two equilibrium solutions. 


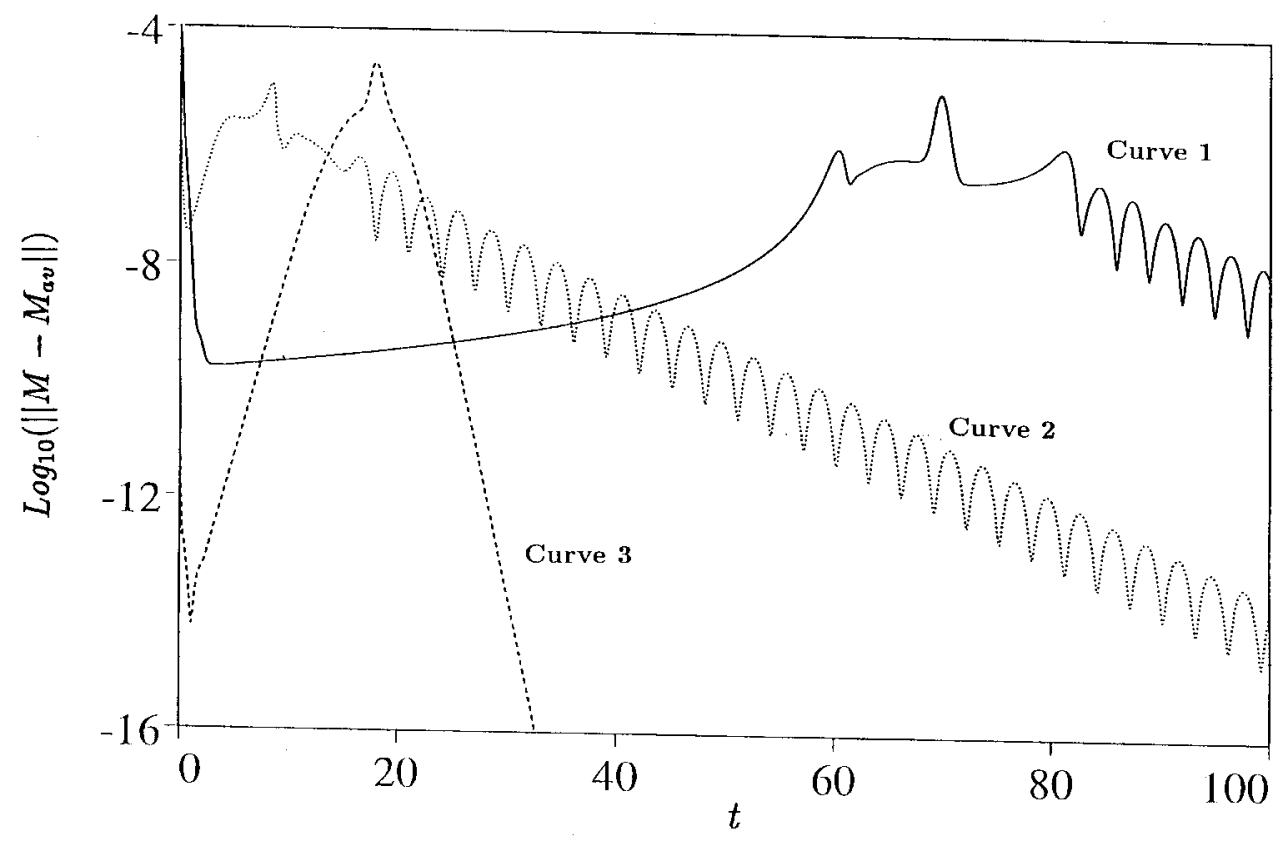

FIG. 13. The convergence history of the solutions of the time-dependent problem from various sets of starting values. Here Curve 1 and Curve 2 represent solutions tending toward the third solution branch, and Curve 3 a solution tending toward the first solution branch.

6. Conclusions. The results of our analysis and of our computations show that the use of an adaptive strategy does not prevent the existence of spurious asymmetric solutions of the discretization of the partial differential equation (1.1). Furthermore, these solutions can be stable while the main symmetric solution branch (which is the approximation to the true solution) is unstable. Hence, they have a profound and misleading effect upon the dynamics of the solution. If we measure the "goodness" of an adaptive strategy by the smallness of the value $\varepsilon_{1}$ of $\varepsilon$ at which the first spurious branch of solutions bifurcates from the main branch, then it would seem that, using a uniform mesh (with $\alpha=0$ ), we do rather better than taking an adaptive mesh with almost any value of $\alpha<1$. Presumably, this is because the adaptive methods reduce the density of mesh points close to the boundaries where we predict, from considerations of supersensitivity, that important features of the solution need to be resolved. We conclude that an adaptive strategy for problems of the form (1.1) which concentrates on significant features such as the transition layer but ignores subtle effects at the boundaries can give rise to most misleading results.

\section{REFERENCES}

[1] C. Budd, W. Huang, And R. D. Russell, Moving mesh methods for problems with blow-up, SIAM J. Sci. Stat. Comp., 17 (1996), pp. 305-327.

[2] C. Budd, G. P. Koomullil, and A. M. Stuart, On the Solution of Convection-Diffusion Boundary Value Problems by Grid Adaption, University of Bristol Report AM 95-04, UK, 1995.

[3] J. Carr and R. L. Pego, Invariant manifolds for metastable patterns in $u_{t}=\varepsilon^{2} u_{x x}-f(u)$, Proc. Roy. Soc. Edinburgh, 116A (1990), pp. 133-160. 
[4] G. F. Carey and H. T. Dinh, Grading functions and mesh redistribution, SIAM J. Numer. Anal., 22 (1985), pp. 1028-1040.

[5] E. Doedel, AUTO: Software for Continuation and Bifurcation Problems in Ordinary Differential Equations, Cal. Tech. Report, Pasadena, CA, 1986.

[6] C. M. Elliot and A. M. Stuart, The global dynamics of discrete semilinear parabolic equations, SIAM J. Numer. Anal., 30 (1993), pp. 1622-1663.

[7] M. Golubitsky, I. Stewart, and D. G. Schaeffer, Singularities and Groups in Bifurcation Theory, Springer-Verlag, New York, 1988.

[8] W. Huang, Y. Ren, and R. D. Russell, Moving mesh partial differential equations (MMPDEs) based on the equidistribution principle, SIAM J. Numer. Anal., 31 (1994), pp. 709730.

[9] J. G. L. Laforgue and R. E. O'Malley, Viscous shock motion for advection diffusion equations, Stud. Appl. Math., 95, (1995), pp. 147-170.

[10] J. G. L. LAforgue And R. E. O'MALley, Shock layer movement for Burgers equation, SIAM J. Appl. Math., 55 (1995), pp. 332-347.

[11] J.-Y. LEE AND M. J. WARD, On the asymptotic and numerical analyses of exponentially illconditioned singularly perturbed BVPs, Stud. Appl. Math., 94 (1995), pp. 271-326.

[12] V. Pereyra and E. G. Sewell, Mesh selection for discrete solution of boundary value problems in ordinary differential equations, Numer. Math., 23 (1975), pp. 261-268.

[13] Y. Ren AND R. D. Russell, Moving mesh techniques based upon equidistribution, and their stability, SIAM J. Sci. Stat. Comput., 13 (1992), pp. 1265-1286.

[14] R. D. Russell and J. Christiansen, Adaptive mesh selection strategies for solving boundary value problems, SIAM J. Numer. Anal., 15 (1978), pp. 59-80.

[15] G. R. Shubin, A. B. Stephens, and H. M. Glaz, Steady shock tracking and Newton's method applied to one-dimensional duct flow, J. Comp. Phys., 39 (1981), pp. 364-374.

[16] A. B. Stephens And G. R. Shubin, Multiple solutions and bifurcation of finite difference approximations to some steady problems of fluid dynamics, SIAM J. Sci. Stat. Comput., 2 (1981), pp. 404-415.

[17] P. K. Sweby and H. C. Yee, On the dynamics of some grid-adaptation schemes, Proceedings of the 4th International Conference on Numerical Grid Generation in CFD and Related Fields, Swansea, UK, 1994.

[18] J. F. Thompson, A Survey of Dynamically-Adaptive Grids in the Numerical Solution of Partial Differential Equations, AIAA Paper, AIAA-84-1606, 1984.

[19] M. J. WARD AND L. G. ReYNA, Internal layers, small eigenvalues and the sensitivity of metastable motion, SIAM J. Appl. Math., 55 (1995), pp. 425-445.

[20] A. B. White, JR., On selection of equi-distributing meshes for two-point boundary-value problems, SIAM J. Numer. Anal., 16 (1979), pp. 472-502. 